\title{
Nonfractional Crystallization of a Terrestrial Magma Ocean
}

\author{
Viatcheslav S. Solomatov and David J. Stevenson
}

Division of Geological and Planetary Sciences, California Institute of Technology, Pasadena

\begin{abstract}
It has been suggested that evolution of a terrestrial magma ocean does not unavoidably follow a fractional crystallization scenario. Convection is able to preclude differentiation until a sharp viscosity increase occurs near some critical crystal fraction. However, this kind of crystallization and its physical and chemical consequences have not been previously studied. We consider an end-member, called here nonfractional crystallization. We begin with a simple equilibrium thermodynamical model of partial melts which is based on an ideal three-component phase diagram. It allows a self-consistent calculation of physical and chemical parameters in the melting range at all interesting pressures. In particular, adiabats of the convecting magma ocean are calculated. The sharp increase in the viscosity is supposed to occur near the maximum packing crystal fraction. However, almost independently of this value, convection occurs even in the highly viscous quasisolid part of the magma ocean and it is strong enough to prevent differentiation in deep regions. A kind of compositional convection occurs due to the layered differentiation, although it is weaker than the thermal convection. Only a surface region undergoes an essential differentiation via melt expulsion by compaction. The thickness of this layer depends on the rheology of partial melts, critical crystal fraction, and crystal sizes but in any case the basal pressure hardly can exceed 5 - $10 \mathrm{GPa}$. Because of lower pressures in the Moon, the thickness of the differentiating layer is large and thus the entire lunar magma ocean could undergo a strong differentiation. Remelting due to the energy released by differentiation is crucial only for much deeper layers (possibly deeper than about $1000 \mathrm{~km}$ for the Earth). For the remaining shallow layer $(p<5-10 \mathrm{GPa})$ the predicted increase of the melt fraction is less than $40 \%$ at the surface and decreases to zero at the bottom of the differentiating layer. Thus, the nonfractional crystallization is suggested to be a likely alternative to the fractional crystallization. The crucial and still poorly understood factors are suspension in convective layers, rheology of partial melts, crystal size, and surface conditions. The most pronounced chemical consequence of the nonfractional crystallization is an almost completely preserved undifferentiated lower mantle and possibly a significant undifferentiated part of the upper mantle. At all depths, in the beginning of differentiation not only the first liquidus solid phase but also subsequent phases have been partially crystallized. So, when the differentiation begins, it involves mixtures of phases. It is important for the remaining layer where differentiation is unavoidable: this layer does not have as strong differentiation of minor elements as in the case of fractional crystallization but it will still involve differentiation of major elements. Future geochemical calculations of this multiphase differentiation, considering both major and minor elements, could help to constrain the differentiation further.
\end{abstract}

\section{INTRODUCTION}

No one doubts that the Earth underwent some differentiation, but the way in which it happened and the results of this differentiation are subjects of intensive debates. The hypothesis of a terrestrial magma ocean in the early history of the Earth is an attractive way to get final or almost final differentiation of the Earth in one stage. So far, differentiation of a terrestrial magma ocean was studied mostly with the help of geochemical approaches. Several models have been suggested. Ohtani et al. [1986], Ohtani and Sawamoto [1987], Herzberg and Gasparik [1991], and Herzberg [1992] suggest that the peridotite upper mantle can be a result of majorite fractionation. Agee and Walker [1988] argue for a subtraction of perovskite and addition of olivine in a chondritic Earth. Similar calculations by Ito and Takahashi [1987], and Takahashi and Ito [1990] suggest a small perovskite fractionation. Studies by Kato et al. [1988a,b], Ringwood [1990], McFarlane and Drake [1990], Drake et al. [1992] and McFarlane et al. [1992] show that fractionation of major components causes an extreme fractionation of mi-

Copyright 1993 by the American Geophysical Union.

Paper number 92JE02579.

0148-0227/93/92JE-02579\$05.00 nor elements. Such a fractionation would be inconsistent with geochemical data. However, Murthy [1991, 1992] and Jones et al. [1992] show that the partition coefficients used in minor element calculations could be quite different at high temperatures and require more accurate analysis. Moreover, Walker and Agee [1989] argue that the experiments on partition coefficients can be influenced by nonequilibrium effects and showed that the perovskite fractionation hypothesis could be consistent with both major and minor element data.

In addition, the evolution of the Earth after the magma ocean period can be also an important part of the entire history of differentiation [e.g., Zindler and Hart, 1986].

As Taylor and Norman [1992] summarize, the Earth might never have had a magma ocean, or the magma ocean somehow avoided stratification, or the Earth was rehomogenized, or the initial composition of the Earth was different from what we usually assume. And, of course, all these factors together with still unrecognized ones, can mix in unknown proportions.

Investigation of the physics of magma oceans can provide additional constraints. Tonks and Melosh [1990] argue that the turbulent convection in the magma ocean is able to preclude crystals settling. The problem of suspension is developed by Solomatov and Stevenson [this issue (a)], where the fractional crystallization is shown to be avoidable, although some problems such as rheology of partial melts and 
crystal sizes remain to be solved. It is also argued that if the fractionation does not take place at the liquidus, then crystallization without differentiation proceeds further until a sharp viscosity transition occurs.

Neither the work by Tonks and Melosh [1990] nor by Solomatov and Stevenson [this issue (a)] answer the question on what happens if the crystallization without differentiation is able to reach a large crystal fraction. Tonks and Melosh [1990] find that the rate of crystallization is fast in comparison with the percolation. They conclude that the magma can not escape from the matrix on the time scale of crystallization to $50-60 \%$ and that the magma ocean solidifies in equilibrium. However, after crystallization to $50-60 \%$ of the crystal fraction, the system unavoidably undergoes differentiation via melt percolation. In this case, the important factor is the competition between the differentiation rate and the rate of further crystallization via cooling controlled by high-viscosity convection. Moreover, the energy release upon differentiation can remelt most of the mantle, and a secondary, chemically differentiated global magma ocean can form. So, the problem of what happens if the fractional crystallization has been avoided has not been solved.

$A b e$ [1991, 1992] considers a parameterized model of evolution where differentiation is controlled by competition between sedimentation and turbulent diffusion. The author assumes that the crystals do not influence convection. However, this influence can be crucial [Solomatov and Stevenson, this issue $(a)$ ]. For example, this is the factor which controls whether or not the fractional crystallization is avoidable at all, or whether or not the sedimentation is possible at all. Another problem is that convection and differentiation in a solidlike region (beyond the critical crystal fraction) occur in a laminar regime where the entire concept of turbulent diffusion is inapplicable, and other approaches are required. Convection in the solidlike regions of a deep magma ocean is also quite different: on the magma ocean time scales it is driven not only by the heat flux from the magma ocean but mostly by the difference in slope between the critical crystal fraction temperature curve and the "wet" adiabat, as will be shown in this paper.

The present paper continues the discussion of the physics of a terrestrial magma ocean. We assume that the fractional crystallization does not take place and sedimentation is negligible, and consider consequences of differentiation which starts only when some critical crystal fraction is reached. We develop an analytical self-consistent thermodynamical model of a multicomponent magma ocean in the melting range and place the rheological transition at a critical crystal fraction. The possible range for this parameter is calculated in some simple way suggested for suspensions (see below). This turns out to be not as important as uncertainties in other parameters. The thermodynamical model is used also to find thermodynamical parameters and temperature distribution in the convective magma ocean ("wet" adiabats).

Convection in quasi-solid and solid regions of the magma ocean is estimated. Its role in reducing of differentiation turns out to be crucial. Effects of compositional convection and remelting due to differentiation are also considered.

\section{Thermodynamics of a Magma Ocean}

What is the simplest system with the help of which we can reproduce thermodynamics of the magma ocean in up- per mantle? Miller et al. [1991a,b] parameterized the phase diagram of a magma ocean at small pressures with the help of an assumed solidus and liquidus and an assumed variation of the crystal fraction between these two curves. To understand basic features of more realistic systems, we consider a self-consistent model which is based on the assumption of ideality. This model uses properties of the components and predicts phase and compositional changes, and corresponding changes in multiphase thermodynamical parameters during various kinds of evolution. The upper mantle consists mainly of olivine and pyroxenes. So, we could consider a two-component ideal olivine-pyroxene system. Such a system is a sufficient approximation unless we are too close to the solidus where this system predicts steplike melting at the eutectic point. Pyroxene is responsible for most of the complicated transitions in the mantle and can form a variety of eutecticlike systems or solid solutions [e.g., Fei et al., 1990; Herzberg and Gasparik, 1991]. Thus, to understand the basic thermodynamics near the solidus we consider a more complicated system, assuming that the pyroxene is a complex, low $\mathrm{MgO} / \mathrm{SiO}_{2}$, two-component system. This subsystem will be considered as a eutecticlike system (as, for example, clinopyroxene and orthopyroxene at low pressures) or a solid solution (as, for example, a garnet at high pressures). Although none of these systems is a good approximation at all pressures, these three-component models give us a range of variants.

The lower mantle is believed to be more simple except for a possible phase transition of FeO [Jeanloz and Ahrens, 1980; Knittle and Jeanloz, 1991]. The composition of the lower mantle can be approximated by a mixture of perovskite and magnesiowüstite [e.g., Ohtani, 1985; Ringwood, 1990]. Because most of the iron is in the magnesiowüstite [Bell et al., 1979; Ito and Yamada, 1982], for the phase diagram calculations (but not for density difference calculations) it is reasonable to model the lower mantle as a three-component mixture of perovskite, periclase, and wüstite in which periclase and wüstite form a solid solution. This is a development of a two-component model described by Miller et al. $[1991 a, b]$.

The thermodynamical model of the three-component system follows. First of all, the thermodynamics of magma in the melting range can be described using only two thermodynamical coordinates, e.g., $T$ and $P$, if conditions of the local thermodynamical equilibrium are satisfied as we assume in this paper. Consider the case when two components form a solid solution. In this case we use indices 1 and 2 for the components which form a solid solution and index 3 for the last component. Suppose that the system is ideal and the chemical potentials of the liquid phases are expressed as

$$
\mu_{i}^{l}=\mu_{i}^{l(0)}+R T \ln N_{i}^{l}, \quad i=1,2,3
$$

and the chemical potentials of the solid phases are the following

$$
\begin{gathered}
\mu_{i}^{s}=\mu_{i}^{s(0)}+R T \ln N_{i}^{s}, i=1,2 \\
\mu_{3}^{s}=\mu_{3}^{s(0)}
\end{gathered}
$$

where the superscript $l$ means liquid and $s$ means solid; $\mu_{i}^{(0)}$ is the chemical potential for pure component $i ; N_{i}^{s, l}$ is the mole fraction of the component in the liquid or in the solid:

$$
N_{i}^{s, l}=\frac{n_{i}^{s, l}}{\sum_{i=1}^{3}\left(n_{i}^{s}+n_{i}^{l}\right)},
$$


$n_{i}$ is the mole fraction of the component $i$ in the total system:

$$
\sum_{i=1}^{3}\left(n_{i}^{s}+n_{i}^{l}\right)=1,
$$

$R$ is the gas constant, $T$ is the temperature.

In the case of the eutecticlike system, the chemical potential for each solid phase is

$$
\mu_{\imath}^{s}=\mu_{\imath}^{s(0)} \text {. }
$$

In the closed system,

$$
n_{i}^{s}+n_{i}^{l}=n_{1}, \quad i=1,2,3
$$

where $n_{2}$ is the mole fraction of the component $i$ in the system.

Conditions of thermodynamical equilibrium require

$$
\mu_{i}^{l}=\mu_{i}^{s} \text {. }
$$

We suppose that the thermal capacities at constant pressure are the same for liquid and solid phases of a component and that the entropy changes on melting are constants. The dependences of $n_{\mathrm{a}}^{l}$ on temperature and pressure then form a system of explicit and transcendental algebraic equations.

The Gibbs free energy is written as

$$
G=\sum_{i=1}^{3}\left(n_{i}^{s} \mu_{i}^{s}+n_{\imath}^{l} \mu_{\imath}^{l}\right) .
$$

Equations (1)-(9) completely describe the system, and now we can derive all thermodynamical functions. They are found in the following forms:

$$
\begin{gathered}
v=\bar{v} \\
c_{p}=\overline{c_{p}}+\sum_{i=1}^{3} T_{0} \Delta s_{i}\left(\frac{\partial n_{i}^{l}}{\partial T}\right)_{p} \\
\alpha=\bar{\alpha}+\sum_{i=1}^{3} \frac{\Delta v_{i}}{\bar{v}}\left(\frac{\partial n_{i}^{l}}{\partial T}\right)_{p} \\
\beta_{T}=\overline{\beta_{T}}+\sum_{i=1}^{3} \frac{\Delta v_{i}}{\bar{v}}\left(\frac{\partial n_{i}^{l}}{\partial p}\right)_{T} \\
c_{v}=c_{p}-\frac{v T \alpha^{2}}{\beta_{T}} \\
\beta_{s}=\beta_{T} \frac{c_{v}}{c_{p}}
\end{gathered}
$$

where $v$ is the molar volume of the system, $c_{p}$ and $c_{v}$ are the heat capacities per mole at constant pressure and volume, $\alpha$ is the coefficient of thermal expansion, $\beta_{T}$ and $\beta_{s}$ are the isothermal and adiabatic compressibilities, $\Delta s_{i}$ and $\Delta v_{i}$ are the molar entropy and molar volume changes on melting, $\bar{v}, \overline{c_{p}}, \bar{\alpha}, \overline{\beta_{T}}$ are the average parameters of the mixture determined by the equations

$$
\begin{gathered}
\bar{v}=\sum_{i=1}^{3}\left(n_{i}^{s} v_{i}^{s}+n_{i}^{l} v_{i}^{l}\right) \\
\overline{c_{p}}=\sum_{i=1}^{3}\left(n_{i}^{s} c_{p}^{s}+n_{i}^{l} c_{p}^{l}\right)
\end{gathered}
$$

$$
\begin{gathered}
\bar{\alpha}=\sum_{i=1}^{3}\left(n_{\imath}^{s} v_{\mathfrak{\imath}}^{s} \alpha_{i}^{s}+n_{\imath}^{l} v_{i}^{l} \alpha_{i}^{l}\right) \\
\overline{\beta_{T}}=\sum_{i=1}^{3}\left(n_{i}^{s} v_{\imath}^{s} \beta_{T}^{s}+n_{\imath}^{l} v_{i}^{l} \beta_{T_{\imath}}^{l}\right)
\end{gathered}
$$

Because the values of the thermodynamical parameters of the pure components both in liquid and solid states are far less than the multiphase additives, we consider only the simplest approximation. We suppose that $c_{p} \approx c_{v} \approx 3 k_{B}$ per atom ( $k_{B}$ is the Boltzmann constant), $K_{T} \approx K_{S}=\beta_{T}^{-1}$ and assume linear dependence of the Grüneisen parameter on volume [e.g., Anderson, 1974; Boehler and Ramakrishnan, 1980]. The thermal expansion can be written as

$$
\alpha=\frac{\gamma c_{v}}{v K_{T}}=\frac{\gamma_{0} c_{v}}{v_{0} K_{T}}=\frac{\alpha_{0}}{1+\frac{K^{\prime}}{K_{T 0}} p}
$$

or

$$
\alpha=\alpha_{0}\left(\frac{v}{v_{0}}\right)^{K^{\prime}}
$$

because of the approximate relationship

$$
\frac{v}{v_{0}}=\left(1+\frac{K^{\prime}}{K_{T 0}} p\right)^{\frac{1}{K^{\prime}}}
$$

where the subscript zero refers to zero pressure and $K^{\prime}$ is the derivative of $K$ with respect to pressure evaluated at zero pressure. The same dependence is assumed for both solids and liquids.

The dependence (21) is exactly the same obtained by Chopelas and Boehler [1989]. They found experimentally the power law with the exponent $5-6$, which is close to the typical values of $K^{\prime}$ for solids and liquids [see also Anderson, 1989].

The volume changes on melting are connected with the melting curve slopes as

$$
\Delta v_{i}=\Delta s_{i} \frac{d T_{0}}{d p}
$$

where $T_{0 i}(p)$ is the melting temperature of the component $i$. The entropy change on melting is usually a very weak function of pressure [Stishov, 1988], and we assume that it is constant.

The final analytical description of the above models consists of a simple but rather bulky system of transcendental equations, and their details will not be written here. However, it is necessary to explain which parameters are important for the calculations we are interested in. In the ideal model we consider, the essential parameters are molecular fractions of the components, their melting temperatures and entropy changes on melting. Together with thermodynamical properties of the single components, these parameters completely describe the system. It is a well-known fact that the exact chemistry does not play any role in ideal systems [e.g., Landau and Lifshitz, 1980]. Moreover, the molecular weight, the entropy change on melting, and the melting temperature are important mostly in combination. For example, almost the same result is obtained if we simultaneously increase the melting temperature of a component and decrease its molecular fraction. These effects are obvious in a linear approximation where all parameters are combined into one controlling nondimensional parameter. 


\section{Thermodynamics of the Upper Mantle}

We suppose that the thermodynamical properties of the both pyroxene components are close to that of enstatite (Table 1) but the melting temperatures are different.

The model is constrained as follows. At zero and small pressures $(<1.5 \mathrm{GPa})$ the model must fit the data compiled by McKenzie and Bickle [1988]. At higher pressures, experiments with peridotites [Takahashi and Scarfe, 1985; Scarfe and Takahashi, 1986; Ito and Takahashi, 1987] and with chondrites [Ohtani and Sawamoto, 1987] were used to constrain the liquidus and solidus and to get the liquidus phase change around $13-15 \mathrm{GPa}$. The difference between chondrites and peridotites is not so essential for our purposes, although our model clearly shows that extracting of low $\mathrm{MgO} / \mathrm{SiO}_{2}$ components shifts the liquidus phase change to higher pressures, in accordance with Ohtani [1990]. We fit the data with the help of the melting curves of the components.

The results of this fit are shown in Figures 1, 2, and 3. Calculations of thermodynamical parameters at zero pressure and the adiabats in the upper mantle pressure range for the solid solution case are also shown.

The thermodynamical behavior in partially molten regions has a simple explanation. First of all, all normalized parameters are equal to 1 out of partially molten regions (when the system is completely solid or completely liquid). Crystallization of a solid phase causes a steplike increase in the thermodynamical parameters. Thermal capacity, for example, increases due to release of the latent heat, thermal expansion or compressibility, due to the volume change upon melting and so on. Not the total amount of the new phase but the rate of its change with temperature or pressure is important. This is the reason for a steplike behavior at the onset of a new phase. The slope of the adiabats is correspondingly changed at these phase boundaries.

Significant qualitative and quantitative differences between the eutecticlike system and the system with a solid solution are as follows. In the first case, the system exhibits a steplike melting at the solidus (usual eutectic melting) but in the case with a solid solution the melting is continuous. The increase in the thermodynamical parameters due to the crystallization of the second phase is within one order of magnitude or so in the case of eutecticlike system but can be larger in the second case. Depending on the solid solution parameters, this increase can be arbitrary large. In the limit when the solution forming components are essentially similar, the solid solution is reduced to an effectively single component, and the entire three-component system is reduced to a simple two-component eutectic system. In

TABLE 1. Thermodynamical properties of pyroxene and olivine (data from Ohtani [1983])

\begin{tabular}{lcc}
\hline & Pyroxene & Olivine \\
\hline$T_{0 i} \mathrm{~K}$ & 1830 & 2163 \\
$\Delta s / R$ & 4.0 & 6.8 \\
$c_{v} / R$ & 15 & 21 \\
$\mu, \mathrm{g} \mathrm{mol}^{-1}$ & 100 & 140 \\
$d T_{0 \imath} / d P, \mathrm{~K} \mathrm{GPa}^{-1}$ & 128 & 41 \\
$v^{s}, \mathrm{~cm}^{3} \mathrm{~mole}^{-1}$ & 31.44 & 43.79 \\
$\alpha^{s}, 10^{-5} \mathrm{~K}^{-1}$ & $1.4+$ & $3.1+$ \\
& $2.50 \times 10^{-3} T$ & $0.85 \times 10^{-3} T$ \\
$K_{T}^{s}, \mathrm{GPa}^{l}, 10^{-5} \mathrm{~K}^{-1}$ & 103.5 & 125.4 \\
$\alpha^{l}, \mathrm{GPa}^{l}$ & 6.4 & 9.5 \\
\hline
\end{tabular}

this limit, all thermodynamical parameters increase to infinity within the interval (which reduces to zero) between the solidus and the temperature of the second phase appearance, a result of steplike melting. In the solid-solution case a turnaround of the curve behavior near the solidus is due to an increased amount of the component with low melting
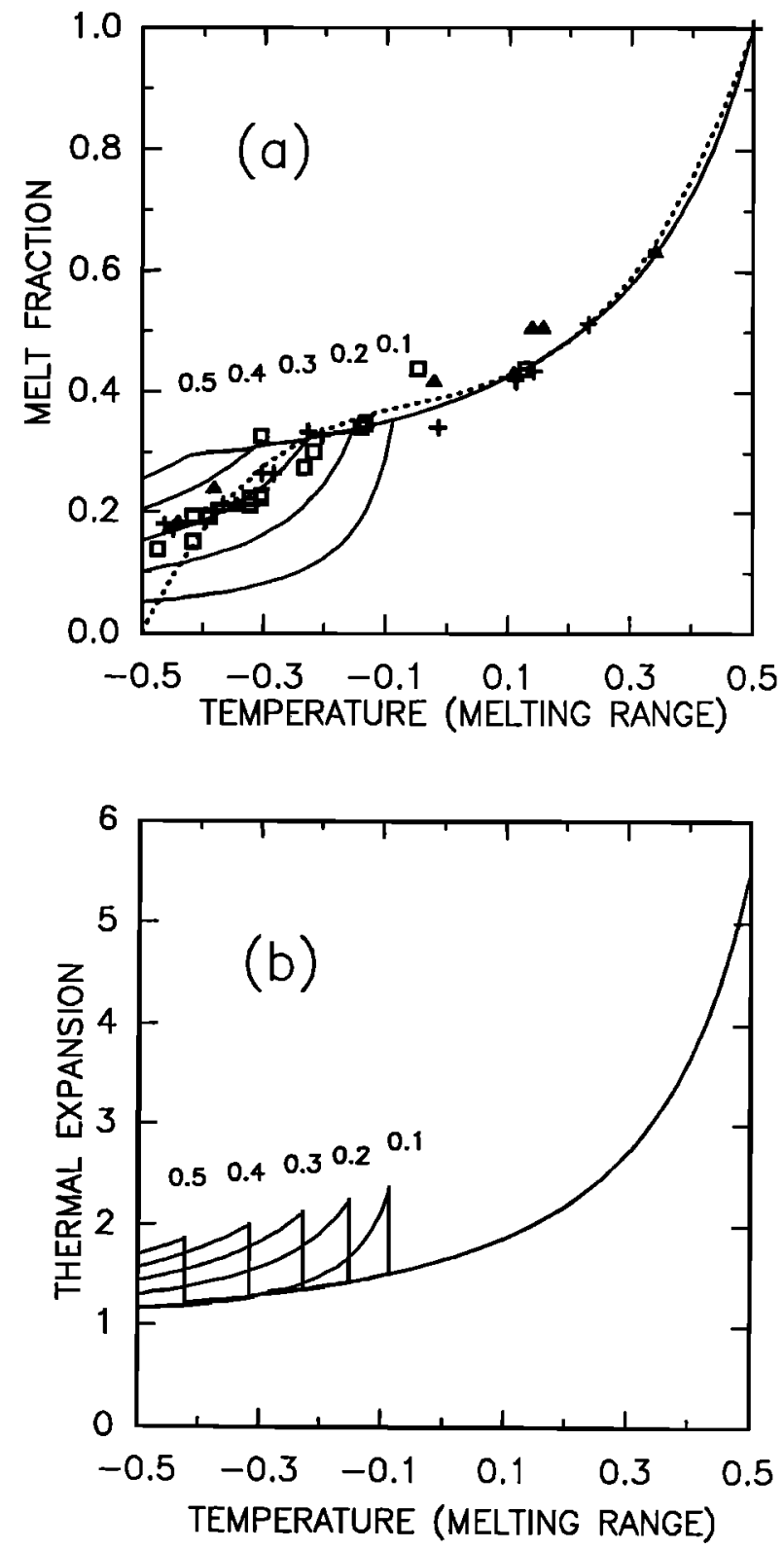

Fig. 1. Thermodynamics of three-component eutecticlike model of peridotite in the melting range. Results of the calculation of $(a)$ weight liquid fraction and the effective thermodynamical parameters of the multiphase systern: (b) thermal expansion, (c) thermal capacity at constant pressure. $(d)$ and isothermal compressibility. The thermodynamic parameters are normalized to the averaged values for the mixture $\left(\overline{c_{p}}, \bar{\alpha}, \overline{\beta_{T}}\right.$ of $\left.(17)-(19)\right)$ The temperature is normalized to the temperature difference between the solidus and liquidus temperature and the zero of the temperature lies half way between liquidus and solidus. The molar fraction of olivine is $2 / 3$. The solid curves are labeled by the ratio of the "pyroxene" components which are supposed to have similar properties except for slightly different melting temperatures (1800 and $1900 \mathrm{~K}$ ). In Figure 1 (a) the experimental data at different pressures (crosses, $0 \leq P \leq 0.5 \mathrm{GPa}$; squares, $0.5<P \leq 1.5$; triangles, $1.5<P$ ) and the parameterization curve (dotted line) are from McKenzie and Bickle [1988]. 

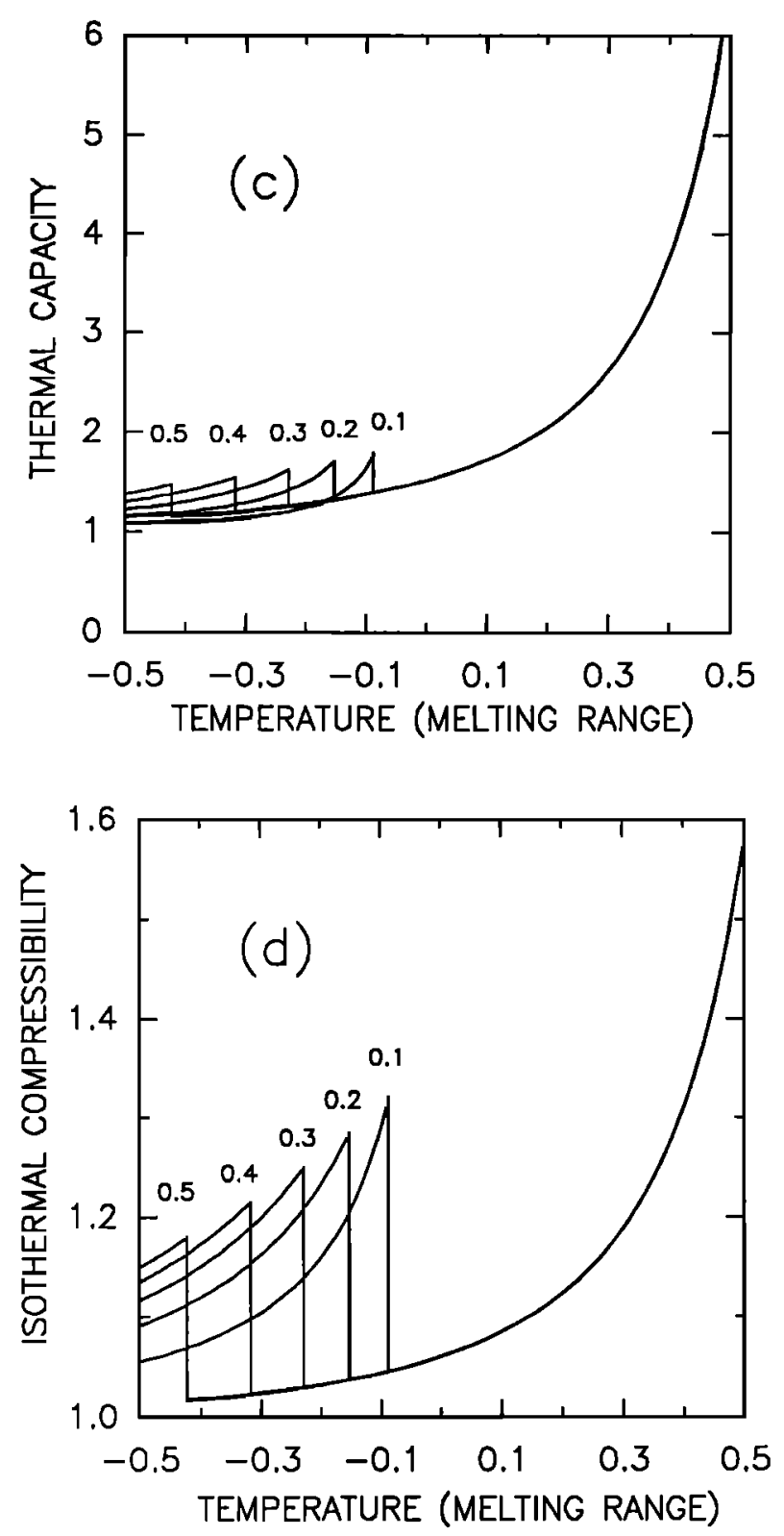

Fig. 1. (continued)

temperature because near this end of the solid solution the rate of change in the amount of crystallizing phase increases with approach to the solidus (second derivative is positive).

At high pressures the adiabats are even steeper than the liquidus in the beginning of solidification and almost parallel to the solidus in the cotectic field. At lower pressures the model gives approximately the same adiabats as those of McKenzie and Bickle [1988].

Although it is hardly possible to expect an agreement between the melting curves used as adjustable parameters with real experimental melting curves, some similarity at low pressures is found for olivine and pyroxene. In the transition region ( $15-25 \mathrm{GPa}$ ), the melting curves used as adjustable parameters are much higher than the experimental melting curves of major minerals [Ohtani, 1983; Scarfe and Takahashi, 1986; Kato and Kumazawa, 1990]. Moreover, the comparison might have no sense at these pressures because of a possible incongruent melting of the olivine [Kato and Kumazawa, 1990].

\section{Thermodynamics of the Lower Mantle}

The lower mantle is supposed to consist mainly of $(\mathrm{Mg}, \mathrm{Fe}) \mathrm{SiO}_{3}$-perovskite and magnesiowüstite, the perovskite being considered as one component (almost pure $\mathrm{MgSiO}_{3}$ ) while magnesiowüstite is a solid solution between periclase and wüstite.

The melting curve of perovskite was experimentally investigated by Heinz and Jeanloz [1987] in the pressure range $25-60 \mathrm{GPa}$ and by Knittle and Jeanloz [1989] up to the pressure $96 \mathrm{GPa}$. Theoretically this curve was estimated by
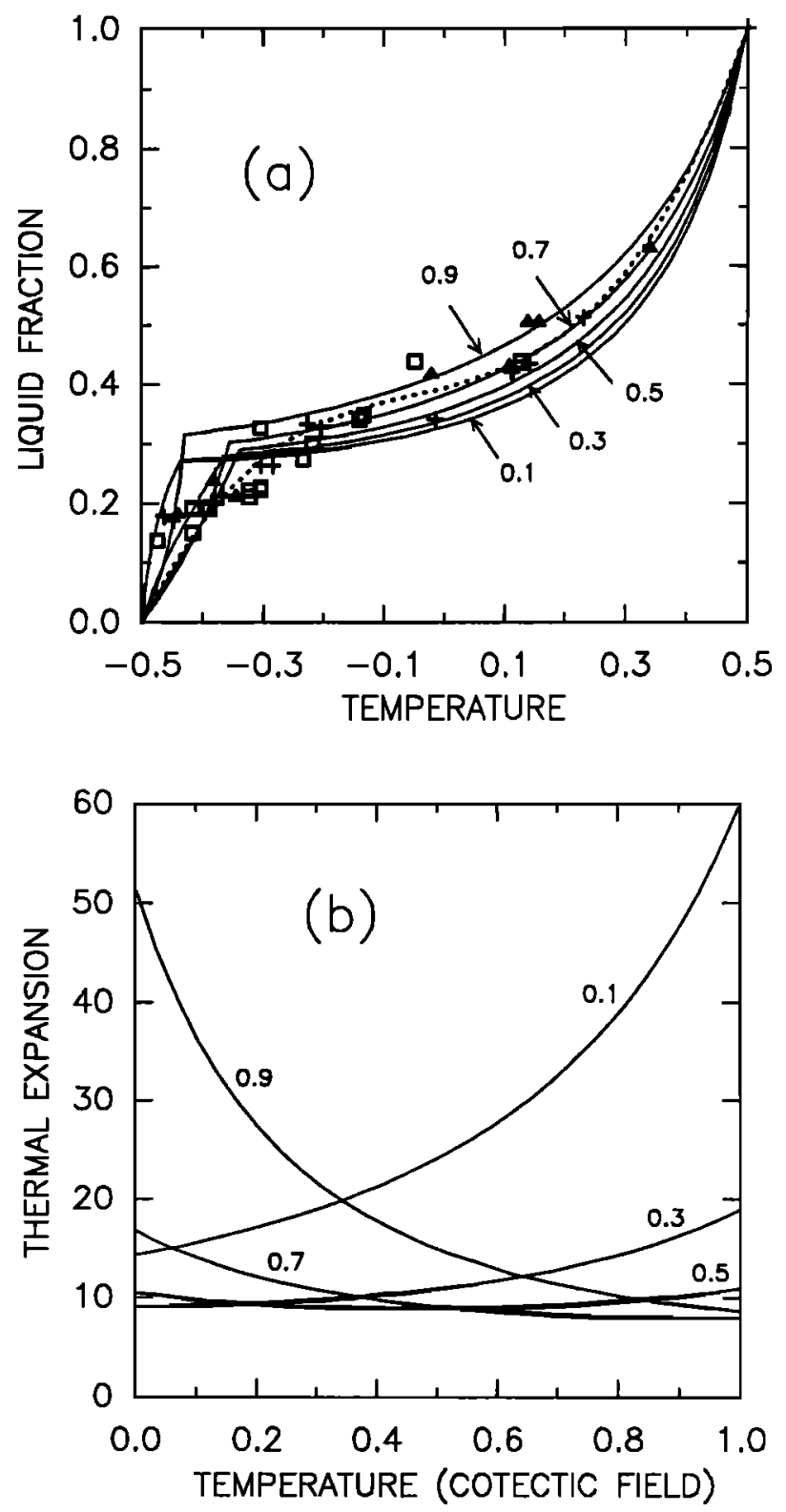

Fig. 2. Three-component model of peridotite with a pyroxene solid solution. Parameters are the same as in Figure 1 but the temperature in Figures $2 b-2 d$ is normalized to the difference between the temperature of the pyroxene appearance and the solidus temperature. The curves are labeled by the molecular ratio of a low-melting temperature component $(1300 \mathrm{~K})$ to a high melting temperature component $(1700 \mathrm{~K})$. The nondimensional temperature " 0 " corresponds to the solidus, and "1" to the temperature of pyroxene appearance. The region between the liquidus and the beginning of the cotectic melting is essentially similar to that of the eutecticlike system. The molar fraction of olivine is again $2 / 3$, and the various curves are labelled as in Figure 1. 

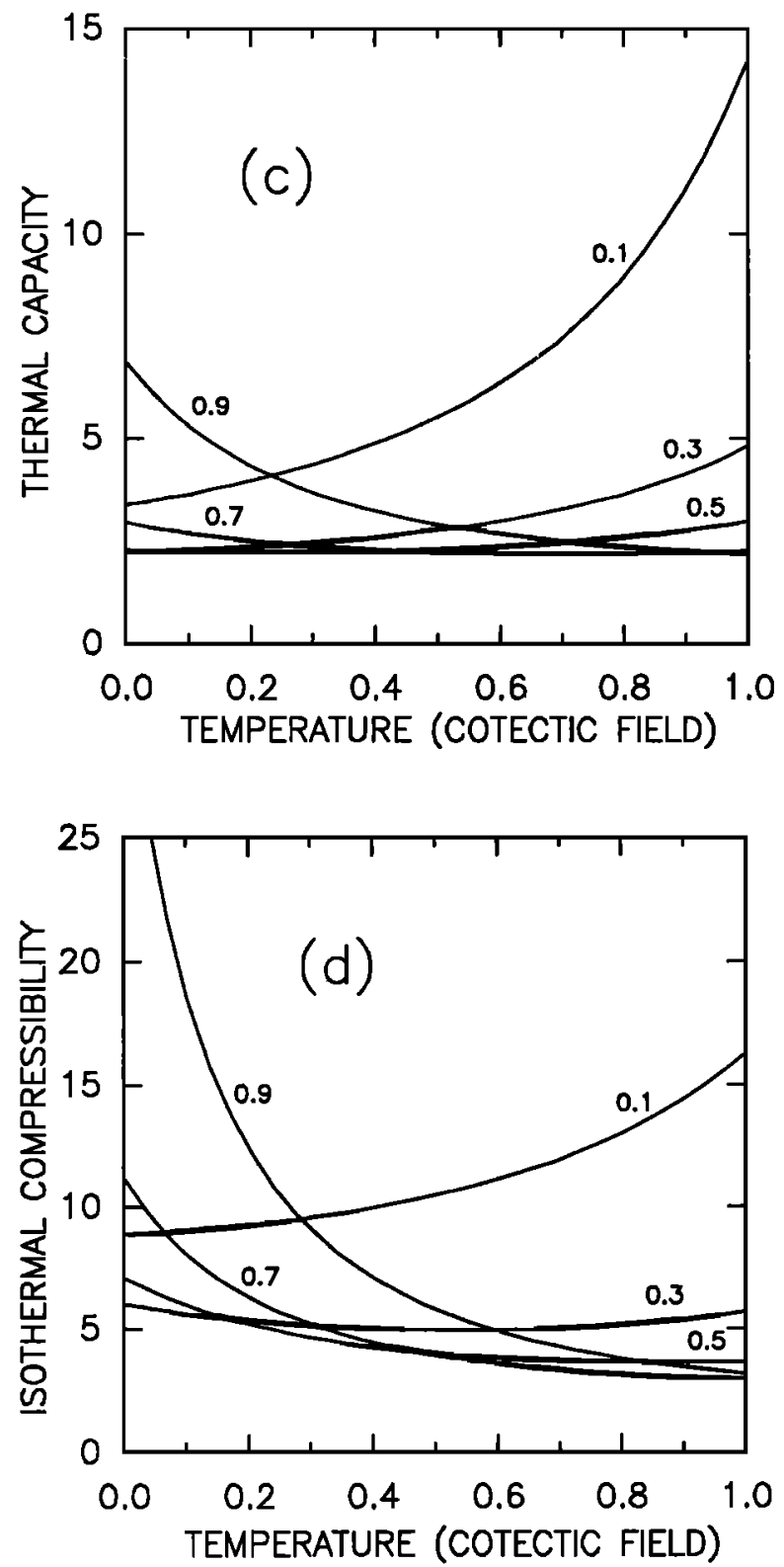

Fig. 2. (continued)

Ohtani [1983] with Kraut-Kennedy and Simon equations, Poirier [1989] with a Lindemann equation, and Stixrude and Bukowinski [1990] with a possibly more fundamental approach based on the thermodynamical properties of both liquid and solid.

We consider two variants of the melting curve for perovskite. The high melting temperature variant is obtained with a Lindemann equation to satisfy experimental data near $25 \mathrm{GPa}$ and is close to the Poirier's curve:

$$
T_{\mathrm{prv}, \mathrm{high}}=2500+26 p-0.052 p^{2}
$$

We assumed that the Grüneisen parameter at zero pressure is equal to 1.73 , the bulk modulus is $266 \mathrm{GPa}$, and its pressure derivative is 3.9 [Knittle et al., 1986; Knittle and Jeanloz, 1987].

The lower melting temperature variant is an approximation to both experimental data of Heinz and Jeanloz [1987], and Knittle and Jeanloz [1989] and theoretical curves predicted by Strixrude and Bukowinski [1990]:

$$
T_{\text {prv,low }}=2500+26 p-0.1 p^{2}
$$

For $\mathrm{MgO}$ and $\mathrm{FeO}$ we also consider the upper and lower melting temperature curves. The upper melting temperature curves are close to Ohtani's [1983] curves at high pressures:

$$
\begin{aligned}
& T_{\text {prc,high }}=3098+67 p-0.2 p^{2} \\
& T_{\text {wst,high }}=1648+46 p-0.1 p^{2}
\end{aligned}
$$

The lower temperature curves are found with the help of Lindemann's equation which are also approximated by quadratic polynomials:
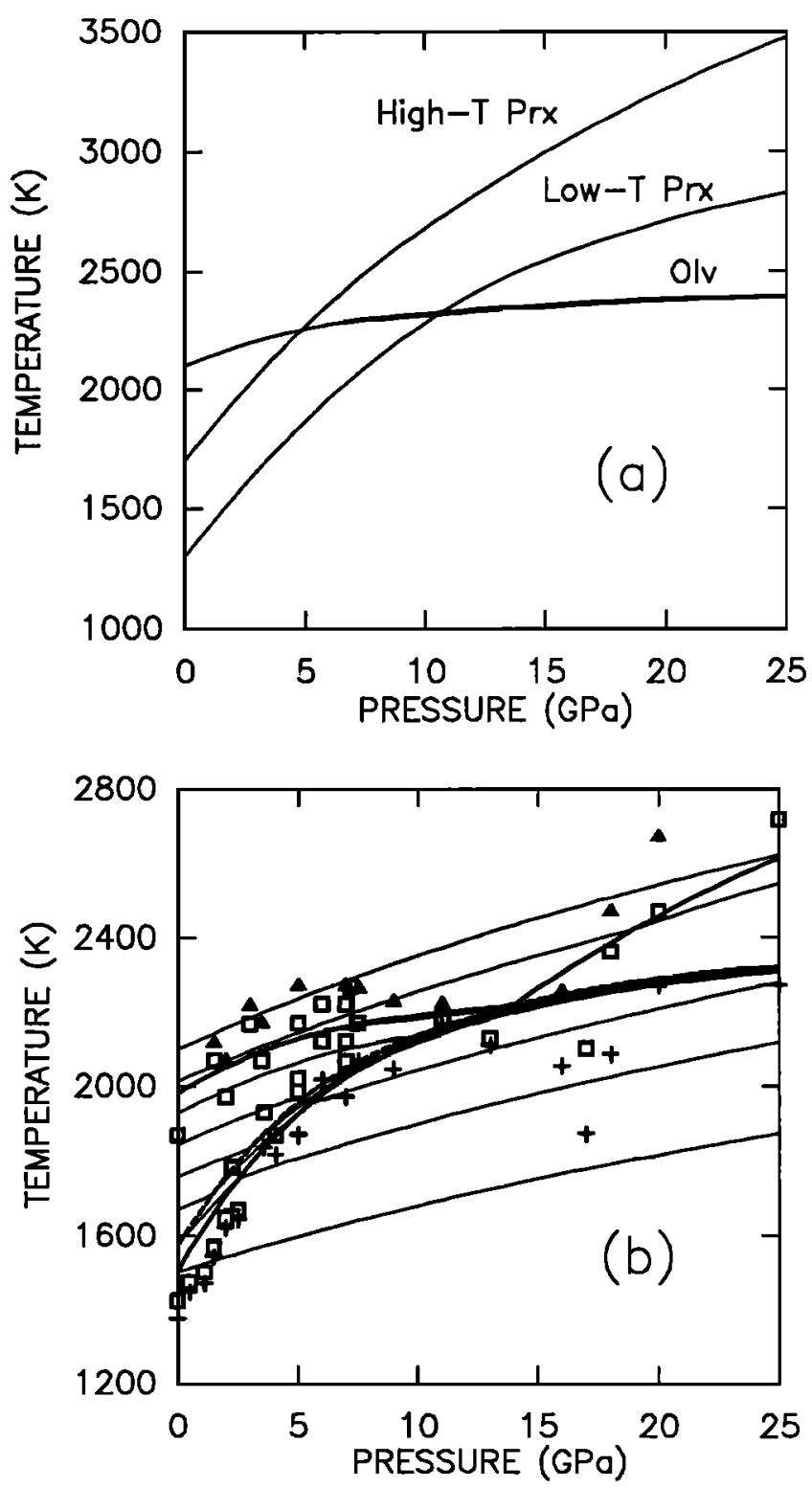

Fig. 3. (a) The melting curves found for olivine and for the "pyroxene" components, which fit the experimental data for the melt fraction (Figure 2), solidus and liquidus. (b) The solidus, liquidus, temperature of the second phase appearance (dashed line) and adiabats are shown for the upper mantle region together with the experimental data for peridotites from McKenzie and Bickle [1988], Scarfe and Takahashi [1986], and Ito and Takahashi [1987]. Liquid region is marked with triangles, solid region with crosses and the partially molten region with squares. The first liquidus phase is olivine at $P<13.7 \mathrm{GPa}$ and garnet (in our simplified model it is, of course, simply a solid solution between low $\mathrm{MgO} / \mathrm{SiO}_{2}$ components of our "pyroxene"-like phase). 


$$
\begin{gathered}
T_{\mathrm{prc}, \text { low }}=3098+37.6 p-0.104 p^{2} \\
T_{\mathrm{wst}, \text { low }}=1648+27 p-0.069 p^{2}
\end{gathered}
$$

where we supposed that for $\mathrm{MgO}$ the Grüneisen parameter at zero pressure is equal to 1.32 , the bulk modulus is 162.7 GPa, its pressure derivative is 4.27 [Anderson et al., 1968]. For $\mathrm{FeO}$, the correspondent values are 1.63 [Jeanloz and Ahrens, 1980], 158, and 4 [Jeanloz and Sato-Sorensen, 1986]. We note that the recent experimental data obtained by Knittle and Jeanloz [1991] for $\mathrm{Fe}_{0.94} \mathrm{O}$ are close to the high-temperature variant for $\mathrm{FeO}$, although Boehler [1992] found lower values that drop the estimates down to our lowtemperature melting curve. The melting curve for $\mathrm{MgO}$ has been recently calculated with the help of molecular dynamics simulations [Gong and Cohen, 1991; Belonoshko, 1992]. At high pressures, it is higher than even the high-temperature curve taken from Ohtani [1983] and requires further justification.

In accordance with the above variants we consider three sets of melting temperatures: $T_{\text {prv,high }}, T_{\text {pre,low }}, T_{\text {wst,low }}$ (model 1), $T_{\text {prv,low }}, T_{\text {prc,low }}, T_{\text {wst,low }}$ (model 2 ), and

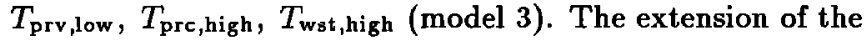
perovskite field (the temperature range between the solidus and cotectics) decreases from model 1 to model 3.

The entropy changes on melting are supposed to be equal to $k_{B}$ per atom, as this is approximately true for simple systems [Stishov, 1988] and also for silicate minerals.

The thermal expansion at zero pressure is calculated using the known values of the Grüneisen parameter at zero pressure and the densities of the components at zero pressures: $3.6,5.9,4.1 \mathrm{~g} \mathrm{~cm}^{-3}$ for periclase, wüstite and perovskite respectively.

Because the lower mantle is simplified to an ideal system consisting of three minerals $\left(\mathrm{MgSiO}_{3}, \mathrm{MgO}\right.$, and $\left.\mathrm{FeO}\right)$ the molar fraction of magnesiowüstite $c_{\mathrm{mw}}$ slightly depends on how we estimate the reduced composition. The value 0.33 corresponds to the unit molecular olivine/pyroxene ratio, 0.29 corresponds to the pyrolite composition [Ringwood, 1970] if we take only $\mathrm{SiO}_{2}, \mathrm{MgO}, \mathrm{FeO}$, and $0.31-0.32$ corresponds to the molar fraction of magnesiowüstite in a chondritic mantle (using estimates from Ringwood [1970] and Ohtani [1983]). So we varied the magnesiowuistite molar fraction in the range $0.25-0.35$, while the ratio $\mathrm{Fe} /(\mathrm{Fe}+\mathrm{Mg})=0.1$ [Ringwood, 1970] was kept constant.

The results of calculations are shown in Figures 4, 5, and 6. From model 1 to model 3 the magnesiowüstite solidification field is extending so much that in the last model it becomes the liquidus phase at some pressure. It is an unavoidable consequence of the high melting temperature of $\mathrm{MgO}$ and to a lesser extent of FeO. In some melting experiments on chondrite or peridotite systems it was difficult to determine what is the first liquidus phase, perovskite or magnesioüstite [Ohtani, 1990]. So, it is possible that the magnesioüstite indeed can substitute perovskite as the first liquidus phase.

In the limit of a very "strong" phase transition, the slope of adiabats would be approximately equal to the slope of the phase boundary. Together with a small difference in slope between the adiabats and the phase boundaries at high pressures, this explains why the partially molten region extends over a large depth interval, which is even larger than the interval predicted by Miller et al. [1991].

In model 2 the solidification of the first liquidus phase be-
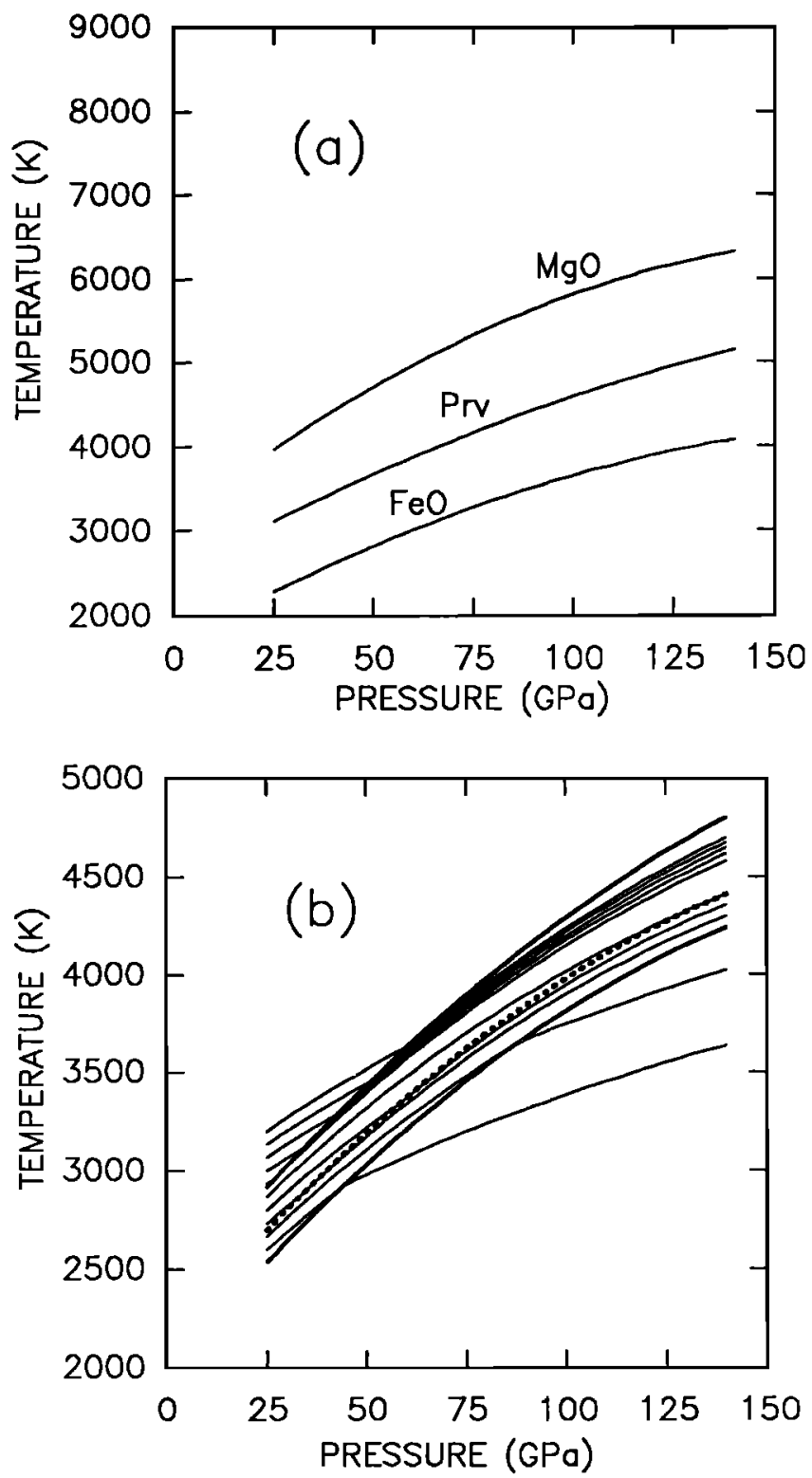

Fig. 4. Model 1: High melting temperature of perovskite and lower melting temperatures of periclase and wüstite. (a) Melting curves for perovskite, periclase and wüstite. (b) The calculated adiabats (solid lines) corresponding to this model. The total molar fraction of magnesiowüstite is 0.30 . Solidus and liquidus curves are shown with heavy solid lines, and the boundary of the second phase appearing (perovskite in this case) is shown with the dotted line. The first liquidus phase is perovskite.

gins not from the bottom but in the point where the adiabat is parallel to the liquidus curve. This unusual behavior is due to a flattening of the perovskite melting curve at high pressures.

\section{BeginNing of Differentiation}

\section{Rheological Boundary}

Calculated adiabats assume a strong convection without differentiation in the entire magma ocean. However the viscosity increases when the temperature drops and at some critical value the suspension ceases and the differentiation begins [Solomatov and Stevenson, this issue (a)]. We first estimate the crystal fraction at which changes in the dynamical regime occur. 

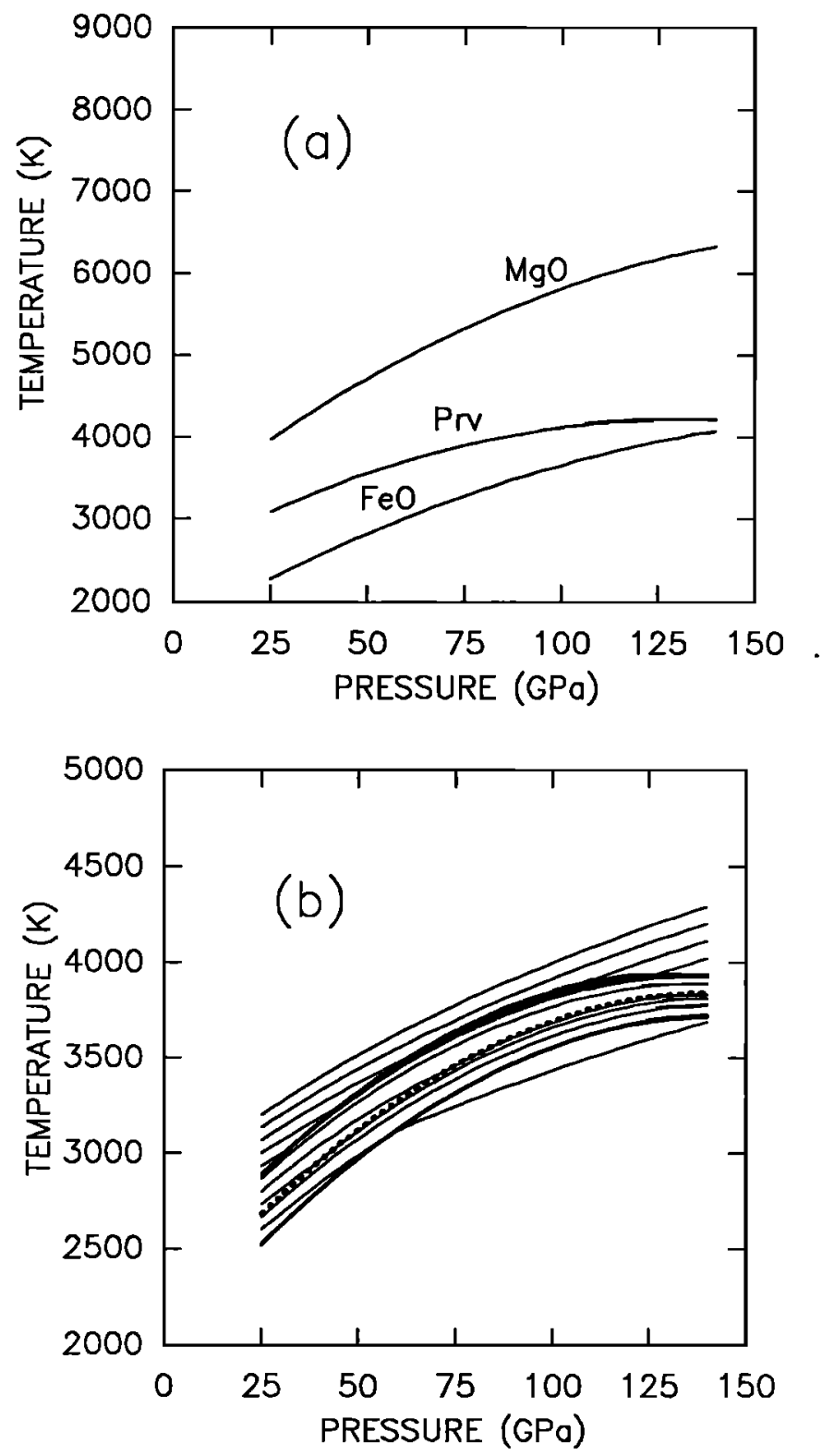

Fig. 5. Model 2: Lower melting temperature of perovskite and lower melting temperatures of periclase and wüstite. Solidification of the first liquidus phase begins not from the bottom but at the level of about $80 \mathrm{GPa}$, where the gradually dropping adiabat of the cooling magma ocean first intersects the liquidus. The first liquidus phase is also perovskite.

The factors that influence the Newtonian viscosity of partial melts are the temperature, pressure, composition, and crystal fraction. Along the liquidus the viscosity is about $10^{-2}-1 \mathrm{P}$ depending on pressure (see discussion by Solomatov and Stevenson [this issue (a)]). The temperature change in the melting range results in variations of the viscosity of less than 1 order of magnitude [e.g., Shaw, 1969]. The composition change of the melt in the melting range increases the viscosity by 2-3 orders of magnitude at small pressures mainly because of the increase in silica fraction during solidification [Bottinga and Weill, 1972; McBirney and Murase, 1984]. This trend in the viscosity change may not be relevant at higher pressures where the first liquidus phase is garnet or perovskite because in this case, silica fraction decreases during crystallization. The above values can be compared with an important convective regime threshold: transition from turbulent to laminar flow. It requires a viscosity increase to $10^{5}-10^{10} \mathrm{P}$ [Solomatov and Stevenson, this issue (a)]. Only crystal fraction change causes the viscosity to increase enormously, from the pure liquid value up to the viscosity of solids $\left(10^{18}-10^{21} \mathrm{P}\right.$ at the solidus). Thus we consider the crystal fraction as the main parameter controlling the Newtonian viscosity.

Models of Newtonian viscosity of concentrated suspensions predict different dependence of the viscosity on the crystal fraction [e.g., Mooney, 1951; Roscoe, 1952; Brinkman, 1952; Krieger and Dougherty, 1959; Murray, 1965; Frankel and Acrivos, 1967; Cambell and Forgacs, 1990]:

$$
\begin{gathered}
\eta \approx \eta_{0}\left(1-\frac{\phi}{\phi_{m}}\right)^{-n} \\
\eta \approx \eta_{0} \frac{\phi / \phi_{m}}{1-\left(\phi / \phi_{m}\right)^{1 / 3}}
\end{gathered}
$$
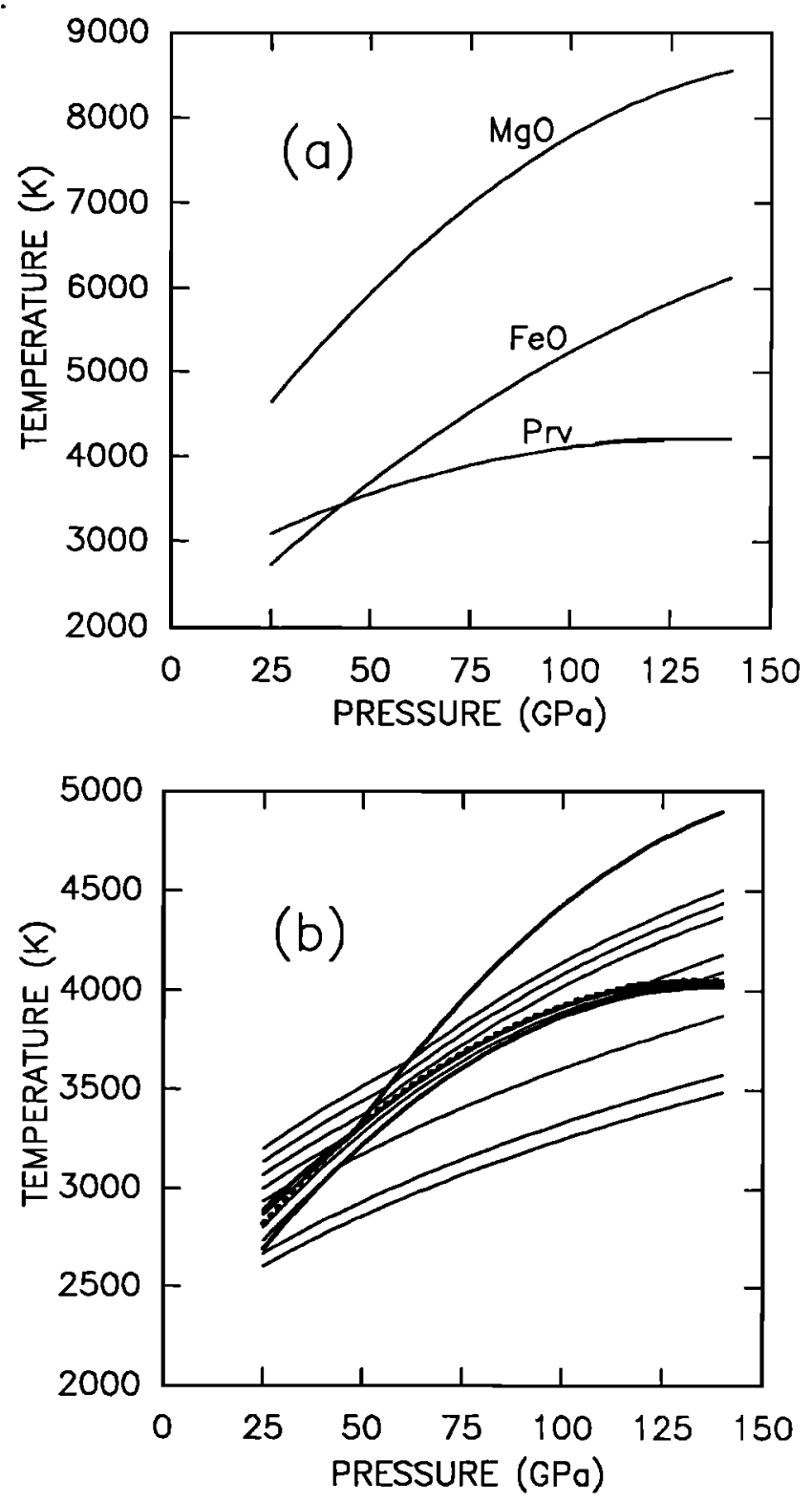

Fig. 6. Model 3: Lower melting temperature of perovskite and high melting temperatures of periclase and wüstite. The first liquidus phase is changing near the $50 \mathrm{GPa}$ from perovskite to magnesiowüstite. 


$$
\begin{gathered}
\eta \approx \eta_{0} \exp \frac{a}{1-\phi / \phi_{m}} \\
\eta \approx \eta_{0} \exp \frac{b \phi}{1-\phi / \phi_{m}},
\end{gathered}
$$

where $a, b, n$ are constants which are approximately equal to $a \approx 1, b \approx 2.5, n \approx 1-3, \eta_{0}$ is the viscosity of pure liquid, and $\phi_{m}$ is the maximum packing volume fraction.

An important feature of all the models is that the viscosity changes significantly only in a small vicinity of the maximum packing solid fraction $\phi_{m}$. The viscosity increase required to change the convective regime from turbulent to laminar is reached when $\phi_{m}-\phi<10^{-2}-10^{-1}$. Thus $\phi_{m}$ effectively determines the transition to quasi-solid viscosity and the beginning of differentiation.

The maximum packing crystal fraction can be calculated independently. It is usually a measured rather than a purely theoretical parameter, and it is defined as a volume fraction of particles which can be reached for a given system. It can be measured in different ways that also require some selection flows [McGeary, 1961; Lee, 1970; Chong et al., 1971; Cumberland and Crawford, 1987]. Cumberland and Crawford [1987] note that the largest change in $\phi_{m}$ takes place on the transition from mono-size to binary mixture. It is natural to consider a binary olivine-pyroxene mixture for the upper mantle and perovskite-magnesiowüstite mixture for the lower mantle. In this case, $\phi_{m}=0.639$ if all crystals have the same radius; the maximum value for a binary mixture consisting of two different sizes is 0.86 . Such an increase is explained by the fact that very small crystals can easily occupy the spaces between large crystals, which effectively increases the total maximum crystal fraction of the system. We assume a spherical shape of the crystals, although in some cases, this can overestimate the maximum packing fraction because any roughness of the surface or deviation from a sphere increases the effective volume occupied by the crystals [Cumberland and Crawford, 1987].

The dependence of the maximum packing solid volume fraction of the binary mixture on the crystal radius ratio and on the fraction ratio in the entire parameter range is calculated with the approach suggested by Lee [1970], and Chong et al. [1971] (both papers considered the viscosity of suspensions) and Cumberland and Crawford [1987] using experimental data of McGeary [1961]. The result is shown in Figure 7.

\section{Composition of Phases in the Beginning of Differentiation}

The position of the boundary between the convective suspension region and the almost immobile solidlike region of packed crystals is determined by intersection between the adiabat which is the averaged temperature curve in the convective magma ocean and the temperature curve along which the crystal fraction is equal to the maximum packing crystal fraction.

The ratio between the velocity $u_{\mathrm{rh}}$ of this boundary (a rheological front) to the melt percolation velocity $u_{\text {perc }}$ is an important parameter that was emphasised by Tonks and Melosh [1990]. It is clear from considering two limiting cases. If $u_{\text {perc }} \gg u_{\mathrm{rh}}$, the melt escapes from the matrix to the lowviscosity region that unavoidably results in changes of the composition of the melt and finally to a strong stratification. If $u_{\text {perc }} \ll u_{\mathrm{rh}}$, the magma ocean solidifies up to some critical crystal fraction without any essential differentiation.

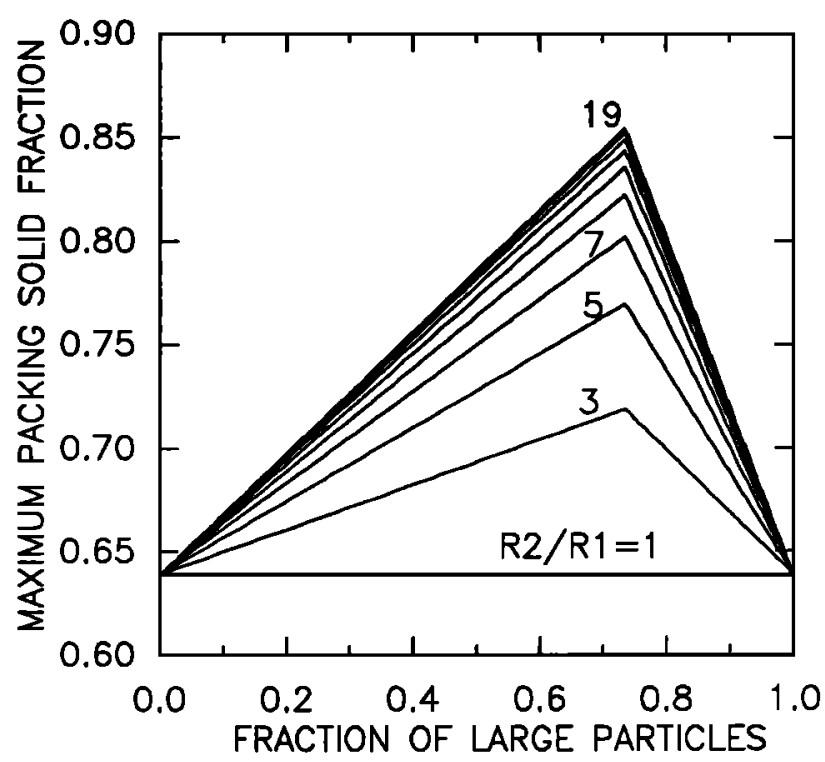

Fig. 7. Maximum packing solid volume fraction for the binary mixture of particles versus the volume fraction of the large particles. The curves are shown for different radius ratios.

Note that the compaction length is very small [McKen$z i e, 1984]$ and the dynamics of differentiation is determined mainly by percolation. The percolation velocity is estimated from the Ergun-Orning formula which is suitable for the melt fractions considered [Soo, 1967; Dullien, 1979]:

$$
u_{\text {perc }}=\frac{4 g \Delta \rho r^{2}(1-\phi)^{2}}{150 \eta t \phi} \approx\left(10^{-\theta}-10^{-4}\right) \mathrm{cm} \mathrm{s}^{-1},
$$

where the crystal radius is equal to $r \approx 10^{-2}-10^{-1} \mathrm{~cm}$ (because we assume nonfractional crystallization, which is valid only if the crystal radius is smaller than $10^{-2}-1 \mathrm{~cm}$; see Solomatov and Stevenson [this issue $(a),(b)])$ and near the critical crystal fraction $\phi_{m} \approx 0.7$ the melt viscosity is $\eta=1-10^{3} \mathrm{P}$ depending on the pressure.

In solidifying of the lower mantle, the velocity $u_{\mathrm{rh}}$ is equal to

$$
u_{\mathrm{rh}} \approx \frac{F A d}{\rho\left(L \phi+c_{p} \Delta T\right) M} \sim\left(10^{-3}-10^{-2}\right) \mathrm{cm} \mathrm{s}^{-1}
$$

where $F \approx 10^{8}-10^{9}$ ergs $\mathrm{cm}^{-2} \mathrm{~s}^{-1}$ is the surface heat flux [Solomatov and Stevenson, this issue $(a)$ ], $A \approx 5.1 \times 10^{18} \mathrm{~cm}^{2}$ is the Earth's surface area, $d \approx 2 \times 10^{8} \mathrm{~cm}$ is the thickness of the lower mantle, $L \approx 10^{10}$ ergs $\mathrm{g}^{-1}$ is the latent heat of solidification (it is approximately proportional to the temperature and about 2 times larger than that one at surface conditions), and $\phi \sim 0.5$ and $\Delta T \sim 1000 \mathrm{~K}$ are the changes in the total solid fraction and averaged temperature upon the solidification of the lower mantle. Thus, the solidification to the critical crystal fraction is much faster than the differentiation rate, in agreement with the conclusion of Tonks and Melosh [1990].

For the upper region $(p<15-25 \mathrm{GPa})$ the solidification is slower because of the drop in the heat flux and associated drop in cooling rate. Solomatov and Stevenson [this issue (a)] estimate that in the absence of a surface rheological boundary layer the heat flux during solidification of the upper mantle is about $10^{8} \mathrm{erg} \mathrm{cm} \mathrm{cm}^{-2} \mathrm{~s}^{-1}$ for the radiative blackbody and $10^{6}-10^{7} \mathrm{erg} \mathrm{cm}^{-2} \mathrm{~s}^{-1}$ in the case of a steam atmosphere. Formation of a rheological boundary layer due to solidification near the surface would reduce the 
heat flux to $10^{5}-10^{7}$ erg $\mathrm{cm}^{-2} \mathrm{~s}^{-1}$ in both cases. Substituting $F \approx 10^{5}-10^{8}$ erg $\mathrm{cm}^{-2} \mathrm{~s}^{-1}, d \approx 6 \times 10^{7} \mathrm{~cm}$, $L \approx 5 \times 10^{9} \mathrm{erg} \mathrm{g}^{-1}, \phi \approx 0.7$, and $\Delta T \sim 300 \mathrm{~K}$ we find

$$
u_{\mathrm{rh}} \approx\left(10^{-6}-10^{-3}\right) \mathrm{cm} \mathrm{s}^{-1},
$$

which may still be larger than the percolation velocity. The estimates depend on the boundary conditions, on the crystal sizes and on the viscosity of pure melts at high pressures.

These estimates show that the cooling and crystallization up to the limiting crystal fraction are so quick that the planet first crystallizes until this fraction is reached and only after that does differentiation begin. Thus, the calculation of the composition of the melt and solids near the maximum packing crystal fraction can be done assuming that the total composition is not essentially modified during solidification. We calculate the composition of the solids and melt along the curve of the critical solid fraction. The results are summarized in Figure 8 . In the case of solidification of the lower mantle, all models demonstrate that not only perovskite but also magnesiowüstite has mostly solidified before the maximum packing is reached. Other phases, particularly $\mathrm{CaSiO}_{3}$, also can be significantly solidified at this moment. Moreover, if the melting temperature of $\mathrm{MgO}$ is really as high as in the model 3 (Gong and Cohen [1991], and Belonoshko [1992] predict even higher values), then our model shows that magnesiowüstite is the first liquidus phase in most of the lower mantle.

In the case of the solidification of the upper mantle (Figure 9) the maximum packing volume fraction of solids is also reached when the second phase has mostly solidified together with the first phase.

The main result of these calculations is formulated as follows. For the Newtonian model of the rheology of partial

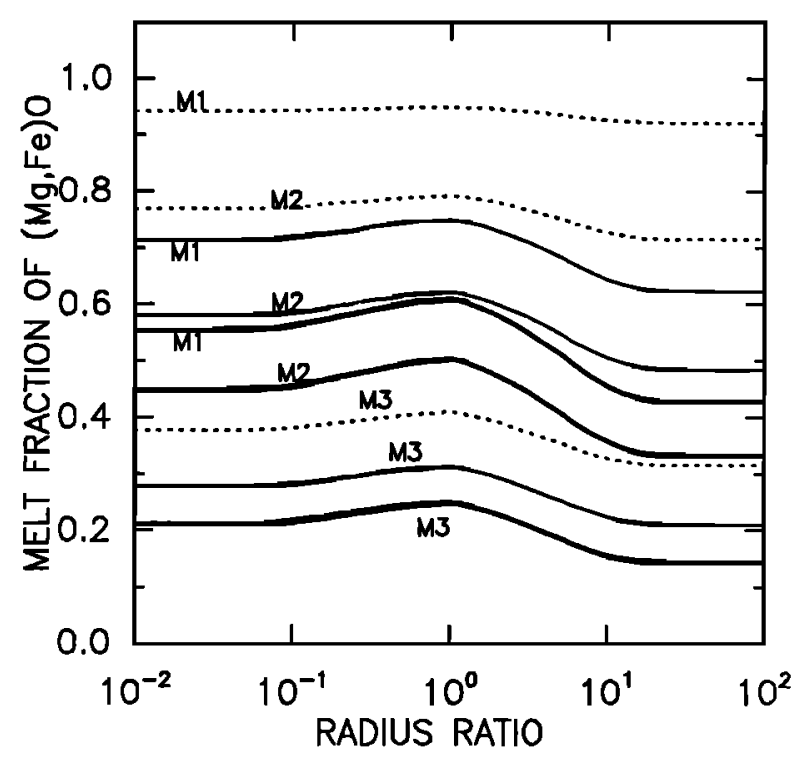

Fig. 8. Lower mantle: amount of magnesiowüstite remaining in the melt (as a ratio to the total amount of magnesiowüstite in the system) at the beginning of differentiation (near the maximum packing crystal fraction) depending on the radius ratio of perovskite crystals to magnesiowüstite crystals. This parameter is averaged over the entire lower mantle pressure range. The curves are shown for different total fractions of magnesiowüstite: 0.25 (dotted lines), 0.30 (thin solid lines), and 0.35 (heavy solid lines) and for different melting curve sets: model 1 (M1), model 2 (M2), and model 3 (M3), presented in Figures 4, 5, 6.

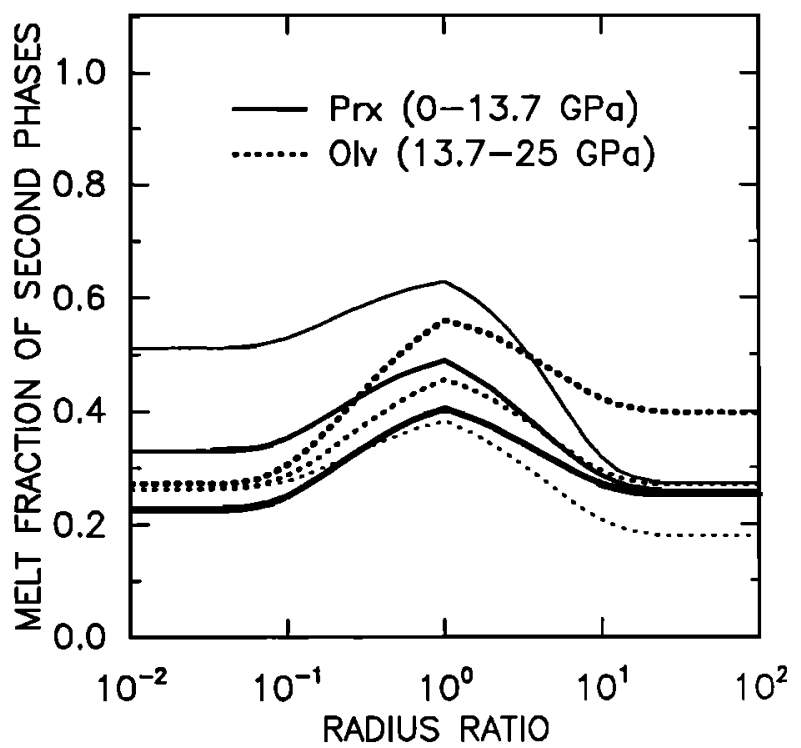

Fig. 9. Upper mantle: The same calculations as for the lower mantle (Figure 8). Because of the crystallization sequence change, the dependence of the amount of the second solid phase remaining in the melt on the olivine/pyroxene radius ratio is shown for two pressure ranges. Solid lines, averaged over the pressure range $0-13.7 \mathrm{GPa}$ (pyroxene is the second liquidus phase); dotted lines, averaged over the pressure range 13.7 - $25 \mathrm{GPa}$ (olivine is the second liquidus phase). The curves are calculated for the total pyroxene fraction $0.3,0.4$, and 0.5 (the thickness of the curves increases correspondingly). This illustrates a dependence on the composition changing from a depleted, peridotitelike system to an undepleted, chondritelike system, correspondently.

melts and for sufficiently small crystal sizes, the differentiation begins (the suspension ceases) when the solid fraction contains different phases, not only the first liquidus phase. Minor element partitioning involves multiphases of this multicomponent solid fraction. This model is qualitatively different from the models used in previous geochemical calculations where one-phase differentiation was considered. However, the beginning of differentiation does not necessary mean that the differentiation can be finished. We show this in the following sections.

\section{Can Solm State Convection Reduce DIFFERENTIATION?}

What is going on in the region beneath the rheological front, which has been solidified to the critical crystal fraction? Is it a dynamically inactive region? Can the trapped melt solidify, or it is differentiated after the solidification of the entire magma ocean to the critical crystal fraction? Certainly it undergoes differentiation. But certainly a solid state convection begins at some moment and provides further cooling and crystallization of the magma ocean. In this section we consider the competition between these two effects.

For simplicity, we ignore the heat flux from the core (which can only help the following arguments) and suppose that at the critical crystal fraction, the viscosity changes from the viscosity of a suspension (which is not so different from the viscosity of pure melt) to the viscosity of solid just near the solidus. This extreme value of the quasi-solid viscosity gives a lower bound for the intensity of the convection beyond the critical crystal fraction. We defined earlier the term rheological front, which is now a sharp boundary between a quasi-liquid and a quasi-solid region, which moves 
up in cooling of the magma ocean. The melt fraction at this transition is still large $(\sim 0.6-0.7)$. The intersection of the averaged temperature curve with solidus will be called the solidification front. The melt is absent at this boundary. In the low-viscosity region, the averaged temperature distribution is almost adiabatic. In the high-viscosity region (below the rheological front) the temperature first follows the critical crystal fraction line which is almost parallel to the solidus.

Figure 10 illustrates the development of convection in this high-viscosity region. The temperature distribution parallel to the solidus is convectively unstable when the thickness of the quasi-solid region exceeds a critical value determined by

$$
\frac{g \beta_{\mathrm{rh}} d_{\mathrm{cr}}^{4}}{\kappa \nu}=R a_{\mathrm{cr}} \sim 10^{3},
$$

where $\kappa$ is the thermal diffusivity, $\nu$ is the kinematic viscosity, and the superadiabatic density gradient $\beta_{\mathrm{rh}}$ is equal to the difference between the gradient of the rheological boundary (line of constant crystal fraction) and the adiabatic gradient:

$$
\begin{gathered}
\beta_{\mathrm{rh}}(d)=\alpha\left[\left(\frac{d T}{d z}\right)_{\mathrm{rh}}-\left(\frac{d T}{d z}\right)_{\mathrm{ad}}\right] \\
\approx \alpha\left[\left(\frac{d T}{d z}\right)_{\mathrm{sol}}-\left(\frac{d T}{d z}\right)_{\mathrm{ad}}\right] .
\end{gathered}
$$

The thermal expansion coefficient $\alpha$ is an effective one for partial melts (see previous sections).

We find

$$
d_{\mathrm{cr}}=\left(\frac{\kappa \nu_{s} R a_{\mathrm{cr}}}{g \bar{\beta}_{\mathrm{rh}}}\right)^{1 / 4}
$$

Substituting $R a_{\mathrm{cr}} \approx 1000$, the thermal diffusivity $\kappa \approx$ $10^{-3} \mathrm{~cm}^{2} \mathrm{~s}^{-1}$, the viscosity of the solid near the solidus $\nu_{s} \approx 10^{18} \mathrm{~cm}^{2} \mathrm{~s}^{-1}$ and $\beta_{\mathrm{rh}} \approx 10^{-9} \mathrm{~cm}^{-1}$ we obtain

$$
d_{c \mathrm{r}} \approx 10\left(\frac{\nu_{s}}{10^{18} \mathrm{~cm}^{2} \mathrm{~s}^{-1}}\right)^{1 / 4} \mathrm{~km} \text {. }
$$

Note that $\kappa=k / \rho c_{p}$, where $k$ is the thermal conductivity, and the superadiabatic gradient $\beta_{\mathrm{rh}}$ are estimated for the multiphase system from the thermodynamical models. Thus when the quasi-solid region is only $10 \mathrm{~km}$ thick, it becomes convectively unstable.

The convective velocities in the quasi-solid region are estimated as follows. At the critical level the convective velocities are about the thermal diffusion rate $\kappa / d \sim 10^{-9} \mathrm{~cm} \mathrm{~s}^{-1}$, which is much smaller than both the velocity of rising of the rheological front and the percolation velocity of the melt. So the quasi-solid region is almost immobile at the beginning of convection. The velocity of the Rayleigh-Taylor instabilities grows with the thickness of the quasi-solid layer $d_{s}$ as [see, e.g., Turcotte and Schubert, 1982]

$$
u_{\mathrm{RT}} \approx 0.1 \frac{\Delta \rho g d_{s}^{2}}{\eta_{s}} \approx 0.1 \frac{\beta_{\mathrm{rh}} g d_{s}^{3}}{\eta_{s}}
$$

The velocities $u_{\mathrm{RT}}$ and $u_{\mathrm{rh}}$ become equal to each other when the thickness of the quasi-solid region is about

$d_{s}=d_{\mathrm{cr}, 2} \sim d_{\mathrm{cr}}\left(\frac{u_{\mathrm{rh}} d_{\mathrm{cr}}}{R a_{\mathrm{cr}} \kappa}\right)^{1 / 3} \sim(100-500)\left(\frac{\nu_{s}}{10^{18} \mathrm{P}}\right)^{1 / 3} \mathrm{~km}$.

The velocity in the quasi-solid region cannot be much larger than the velocity of the rheological front because the quasi-solid region would immediately (in comparison with the rate of the moving of the rheological front) return the system to the equilibrium between these two velocities. Thus the velocity $u_{\mathrm{rh}}$ is a scale for the convective velocity in the quasi-solid region. It is also an estimate for the velocity of the advance of the complete solidification boundary.

An additional important condition must be checked: dissipation energy production per unit volume

$$
\Phi_{\text {diss }} \sim \eta\left(\frac{u_{\text {solid }}}{l_{\text {diss }}}\right)^{2}
$$

in the convecting quasi-solid region must be not larger than the work done by convection per unit volume
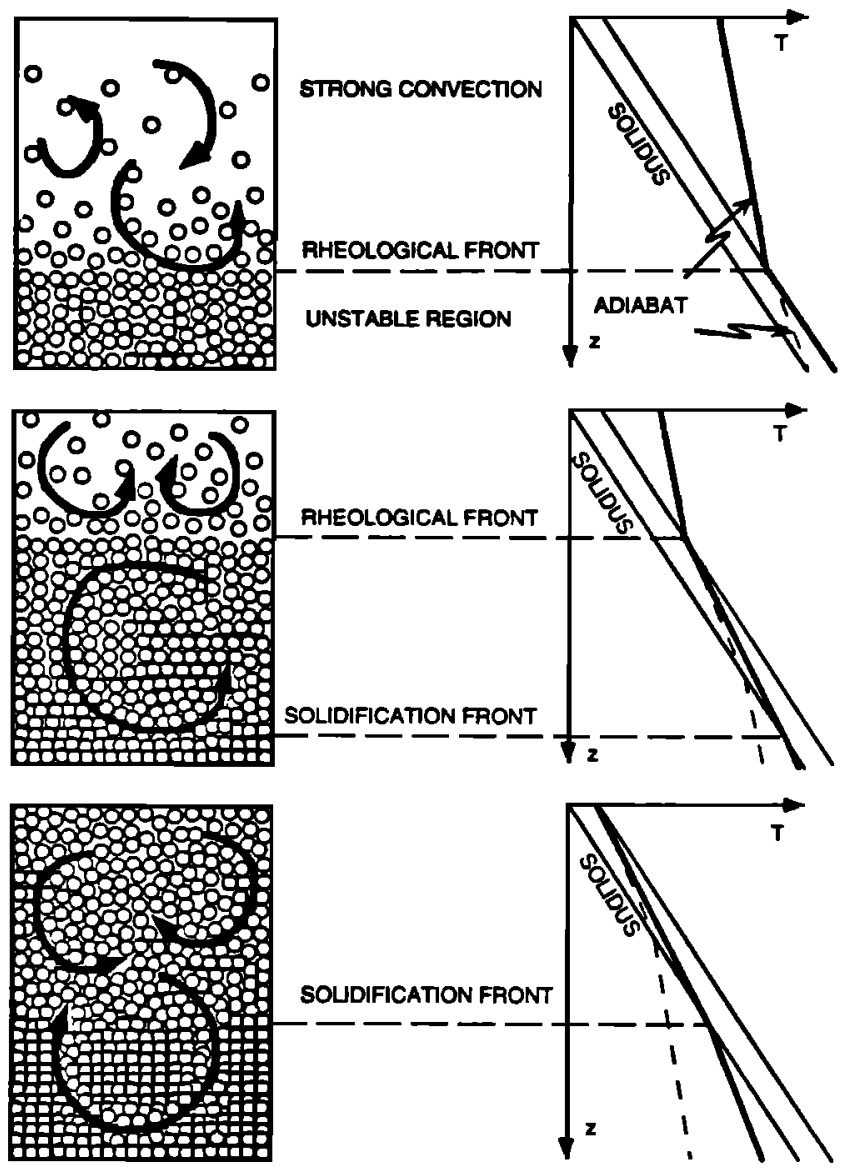

Fig. 10. At some moment the temperature of the convecting magma ocean (heavy solid line) intersects the curve at which the viscosity sharply increases (which is actually a narrow transition region). All dynamical changes occur mostly at that time: suspension ceases, flow becomes laminar, and convection stops. This pressure level is designated as the rheological front. The rheological front rises during cooling. The temperature in the highviscosity nonconvecting region follows this rheological boundary at which the solid fraction is approximately constant. This "constant solid fraction temperature" becomes unstable (top figure) because the slope of this curve is larger than the adiabatic gradient (adiabat is shown by dashed line) and convection begins in the quasi-solid region. The convective velocity reaches the velocity $u_{\mathrm{rh}}$ of the rheological front (see text for details). The temperature curve in the quasi-solid region deviates from the adiabat by a small superadiabatic gradient, which drives the convection and which cannot be larger than some value determined in the text. At some moment the temperature drops below the solidus and a solidification front forms. Eventually the rheological front reaches the surface (bottom figure). At that time the partially molten region is limited by the solidification front. Then the heat flux significantly decreases, the superadiabatic gradient drops, and the convection becomes much weaker, approaching a "normal" solid state convection regime. 


$$
\Phi_{\text {conv }} \sim \frac{\alpha g}{c_{p}} F
$$

The convective length scale $l_{\text {conv }}$ is the characteristic length scale for the velocity changes, which is controlled by the rheology (see some relevant discussion by Solomatov [1993]). We find that

$$
u_{\text {solid }} \sim\left(\frac{\alpha g l_{\text {conv }}^{2} F}{c_{p} \eta}\right)^{1 / 2} .
$$

This is exactly the velocity scale for laminar convection in the layer with the thickness $l_{\text {conv }}$. Substituting $l_{\text {conv }}=10^{7}-$ $10^{8} \mathrm{~cm}$ and $F=10^{5}-10^{8} \mathrm{erg} \mathrm{cm}^{-2} \mathrm{~s}^{-1}$, we find that

$$
u_{\text {solid }} \sim\left(10^{-4}-10^{-1}\right)\left(\frac{10^{18} \mathrm{P}}{\eta}\right)^{1 / 2} \mathrm{~cm} \mathrm{~s}^{-1} \text {. }
$$

When $u_{\text {solid }}<u_{\mathrm{rh}}$ the velocity in the convective region is limited by the scale (46). This would be the case of a solid state convection in the Earth with a heat flux of $10^{5}-10^{6}$ times higher than the present-day heat flux from the Earth. In any case, the convective velocities and the solidification rate are larger than the percolation rate. Thus the temperature in the quasi-solid region drops below the critical crystal fraction line and must approach the calculated equilibrium adiabats even in this quasi-solid or completely solid region.

The important question now is how much the temperature curve deviates from the adiabat. The superadiabatic temperature gradient $d T^{\prime} / d z=d T / d z-(d T / d z)_{\text {ad }}$ connected with the density gradient (parameter $\beta=d(\Delta \rho / \rho) / d z$ ) can be estimated from the requirement that the velocity of the Rayleigh-Taylor instability in the superadiabatic region must be smaller or equal to the convective velocity:

$$
\frac{\beta g l_{\text {conv }}^{3}}{\eta_{s}} \sim u_{r h} .
$$

In the case of usual constant viscosity convection this results in a well-known temperature fluctuation scale away from the thermal boundary layers for laminar convection [Golitsyn, 1978]:

$$
T^{\prime} \sim\left(\frac{\alpha \eta F}{c_{p} g d^{2}}\right)^{1 / 2}
$$

For the problem considered we find

$$
\frac{d T^{\prime} / d z}{(d T / d z)_{\mathrm{rh}}-(d T / d z)_{\mathrm{ad}}} \approx \frac{\beta}{\beta_{\mathrm{rh}}} \sim\left(\frac{d_{\mathrm{cr}, 2}}{l_{\mathrm{conv}}}\right)^{3} .
$$

The moment when the convective velocity becomes smaller than the percolation velocity can be estimated as follows. Consider the last stage of the crystallization, when the rheological front approaches the surface. The heat flux drops by several orders of magnitude within a narrow temperature interval $50-100 \mathrm{~K}$ near the rheological transition [Solomatov and Stevenson, this issue (a)]. When the potential temperature drops below the rheological transition, then the low-viscosity region beneath the surface vanishes. This means that the formation of the thermal boundary layer controlling the heat loss occurs in an effectively solid region, and the heat flux, the cooling rate, the superadiabatic gradient, and the convective velocities decrease by many orders of magnitude at this point. So we assume that the convective velocity becomes smaller than the percolation velocity in a narrow potential temperature range above the temperature of the rheological transition and thus this transition can be approximately considered as an ideal sharp one.
The superadiabatic temperature distribution is calculated at the moment when the convective velocity is about equal to the percolation velocity estimated by its upper bound, at the critical crystal fraction. The superadiabatic gradient is determined by the equation

$$
\frac{\beta g l_{\text {conv }}^{3}}{\eta_{s}} \sim u_{\text {perc }} .
$$

From Fig. 11 we see that it becomes negligible if the crystal radius (it controls $u_{\text {perc }}$ ) is less than $10^{-2}-10^{-1} \mathrm{~cm}$ or so. Thus the "unimpeded" complete melt extraction can take place at pressures smaller than $5-10 \mathrm{GPa}$, an approximate bottom of the layer when this extraction occurs.

In the case of the Moon, this pressure is larger than the pressure in the center of the Moon, and thus the entire lunar magma ocean must undergo differentiation.

The problem depends on various factors, such as the rheology of partial melts (it controls, for example, the rheological transition and the rheology just above the solidus) and the crystal radius or, in general, permeability. Strongly time-dependent convection in this rheologically complicated system and at high Rayleigh numbers also needs further study.

\section{Compositional Convection due to Differentiation}

Let us consider an additional buoyancy effect resulting from the differentiation and working independently on the thermal convection. Suppose that the mantle has been crystallized up to the rheological transition or more. The remaining liquid in the lower mantle is denser than the crystal mixture by about $0.1-0.3 \mathrm{~g} \mathrm{~cm}^{-3}$, depending on pressure, because it is enriched in iron. It is qualitatively consistent with the results by Miller et al. [1991 $a, b]$ (although our model cannot be used for accurate solid-melt density difference calculations). For the upper mantle, in the pressure range between 15 and $25 \mathrm{GPa}$, the garnet-rich crystal mixture is probably denser than the melt, in the pressure range between 8 and $15 \mathrm{GPa}$ the olivine-rich crystal mixture is less dense than the melt, and at pressures less than about 8 GPa they are denser than the surrounding melt [Agee and Walker, 1988]. Thus the partially crystallized magma ocean can have several layers with different and even opposite velocities of percolation.

A simple consequence of this layered differentiation is a compositional convection: when differentiation takes place in the lower layer, the light component rises up and mixes the upper layers. On the other hand the differentiation in an upper layer forms a dense layer at its bottom. It is also gravitationally unstable with respect to the lower layer. The physics of this kind of compositional convection is similar to that was studied experimentally by Huppert et al. [1991]: sedimentation of suspended particles in the lower region forms a differentiated layer which can be lighter than the upper layer. This is the basic mechanism of this kind of compositional convection. To estimate this effect, we note a similarity between different kinds of convection when the driving mechanism is buoyancy forces. In such a case, the important parameter is the buoyancy flux [Golitsyn, 1978]. In the case of a usual thermal convection, the buoyancy flux is equal to

$$
\Phi_{T}=\frac{\alpha F}{c_{p}} .
$$

In the case of differentiation, the buoyancy flux is equal to 

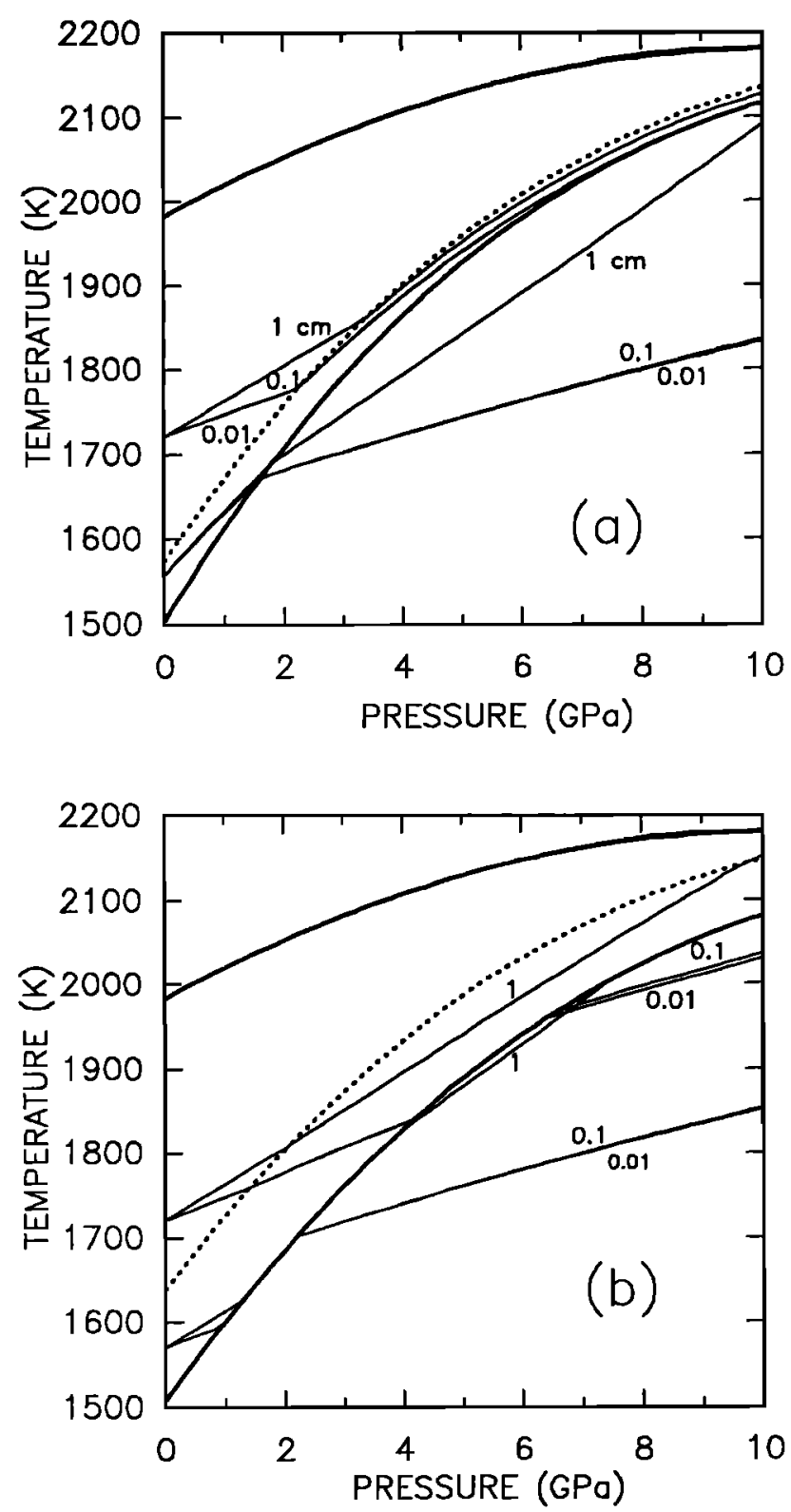

Fig. 11. Calculations of superadiabatic temperature distribution with the condition that the convective velocities in the quasisolid layer are equal to the percolation velocity of the melt: (a) the system with one pyroxene solid solution (as in Figures 2, and 3); (b) the eutectic-like system (as in Figure 1). Two values of the potential temperature chosen correspond to 0.5 and 0.7 crystal fractions. Three values of crystal radius are considered: $10^{-2}, 10^{-1}$, and $1 \mathrm{~cm}$. Only in the last case does the temperature deviate substantially from the adiabat. The ratio between the viscosjty of the solid just near the solidus and the viscosity of the melt is $10^{14}$, the convective length scale is $300 \mathrm{~km}$. Note a step-like melting of the eutectic system (Figure $11 b$ ) at the solidus.

$$
\Phi_{D}=\Delta \rho_{D} u_{\mathrm{perc}} \phi_{l}
$$

where $\Delta \rho_{D}$ is the driving density difference produced by the melt extraction. For example, if we suppose that in a lower layer (e.g., in the lower mantle) the melt moves down then the driving density difference is the difference between the density of the upper layer consisting of the matrix with melt and the density of the matrix of the lower layer after the melt extraction. A compositional boundary layer less dense with respect to the upper layer is formed on the top of the lower layer. It rises up and drives convection in the upper layer. The amplitude of the convective velocities of this compositional convection is

$$
u_{D} \sim\left(\Phi_{D} \frac{g d}{\eta_{s}}\right)^{1 / 2} \sim\left(\Delta \rho_{D} u_{\mathrm{perc}} \phi_{l} \frac{g d}{\eta_{s}}\right)^{1 / 2},
$$

where $d$ and $\eta_{s}$ are the thickness and the viscosity of the upper quasi-solid layer in the considered example.

At low melt fractions the ratio of this velocity to the percolation velocities is about

$$
\frac{u_{D}}{u_{\text {perc }}} \sim\left(\frac{\eta_{l}}{\eta_{s}}\right)^{1 / 2} \frac{d}{r},
$$

which is for $\eta_{l} / \eta_{s} \sim 10^{14}$ and $d / r \sim 10^{9}$ gives the ratio $10^{2}$. So the velocity of this compositional convection can be larger than the percolation velocity. This means that a complicated remixing (in the magma ocean there could be several differentiated layers) takes place at the same time as the differentiation.

The ratio of this velocity to the convective velocities due to the thermal convection is about

$$
\frac{u_{D}}{u_{T}} \sim\left(\frac{\Delta \rho_{D} u_{\text {perc }} \phi_{l} c_{p}}{\alpha F}\right)^{1 / 2}
$$

and for the values of the parameters considered in the previous section is about $10^{-5}-10^{-1} \ll 1$. So the compositional convection is weak and can be ignored in comparison with the solid state convection considered in the previous section.

\section{Remelting due to Melt Extraction}

The differentiation in the remaining surface layer of the mantle releases some energy that produces additional melt and thus increases the extent of differentiation. The released energy consists of a gravitational part and a thermal part. The upper bound for the thermal energy transported by melt is of the same order of magnitude or less than the gravitational energy [see estimates by McKenzie, 1984]. We note that the real value can be significantly smaller: the energy release due to adiabatic upwelling of the melt is essentially compensated by the energy consumption due to adiabatic downward motion of the solid matrix required from the mass conservation condition. The ratio of these two values is proportional to $d_{l} \phi_{l} / d_{s} \phi_{s} \approx 1$, where $d_{l}$ is the adiabatic path of the melt and $d_{s}$ is the adiabatic path of the matrix. In the following estimates we ignore the thermal energy release and consider only the gravitational energy release.

Suppose that the melt fraction is small, the density difference between the solid and the melt is small, and the melt percolates upward. Let us choose the vertical axis $z$ with the zero level at the bottom of the differentiating layer. To estimate the distribution of the released energy with $z$ after the melt extraction, we note that the gravitational energy is transformed to heat through viscous dissipation. Thus at a given level $z$ the energy release between this level and the bottom of the differentiating layer $(z=0)$ is determined by the gravitational energy release $E_{G}\left(z_{1}\right)$ in the interval between 0 and $z$. If the initial melt distribution is $x_{0}(z)$, then per unit area

$$
E_{G}(z)=\Delta \rho g \int_{0}^{z}\left(z-z_{1}\right) x_{0}\left(z_{1}\right) d z_{1} .
$$


This energy is spent on melting:

$$
E_{M}(z)=\rho L \int_{0}^{z} x_{1}\left(z_{1}\right) d z_{1} .
$$

where $L$ is the latent heat and $x_{1}(z)$ is the melt fraction produced by remelting of the solid matrix. Solving the integral equation $E_{G}(z)=E_{M}(z)$, we find

$$
x_{1}(z)=\frac{\Delta \rho g}{\rho L} \int_{0}^{z} x_{0}\left(z_{1}\right) d z_{1} .
$$

For the linear initial melt distribution $x_{0}(z)=x_{0}(d) z / d$, where $d$ is the thickness of the differentiating layer, we obtain

$$
x_{1}(z)=x_{0}(d) \frac{R_{m}}{2}\left(\frac{z}{d}\right)^{2}
$$

where

$$
R_{m}=\frac{\Delta \rho g d}{\rho L} .
$$

Continuing the fractional melt extraction, we find the melt fraction after $n$ extractions

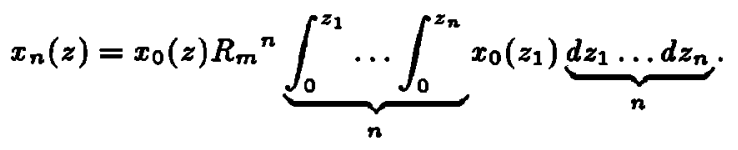

The total melt extraction is equal to

$$
x_{m}(z)=x_{0}(z)+\lim _{n \rightarrow \infty} \sum_{k=1}^{n} x_{k}(z) .
$$

In the case of linear initial melt distribution we find

$$
x_{m}(z)=\frac{x_{0}(d)}{R_{m}}\left[\exp \left(\frac{z}{d} R_{m}\right)-1\right] \approx x_{0}(z)\left[1+\frac{R_{m}}{2} \frac{z}{d}\right]
$$

For $x_{0}(d)=0.3-0.4$ the complete remelting first occurs at the top of the layer $\left(x_{m}(d)=1\right)$ at $R_{m}=1.6-2.1$. This value corresponds to the layer thickness

$$
d_{m} \sim \frac{2 \rho L}{\Delta \rho g} \approx 1 \times 10^{8} \mathrm{~cm}
$$

where we substituted $\Delta \rho / \rho=0.1$ and $L=5 \times 10^{9} \mathrm{ergs}^{-1}$. In the previous sections we showed that the differentiation can involve only a thin layer, possibly less than about 300 $\mathrm{km}$. For such a layer, $R_{m} \sim 0.6$, which corresponds to about $40 \%$ melt fraction increase at the surface. Thus the remaining differentiating layer of the magma ocean can squeeze the melt without any essential remelting although the remelting of the uppermost layers is sensitive to the thickness of the differentiating layer.

\section{Conclusion}

1. An analytical thermodynamical model of a partially molten magma ocean is developed for an ideal system in the approximation of local thermodynamical equilibrium. Adiabats of a convective magma ocean are calculated for the upper and lower mantle.

2. A strong convection occurs even in the quasi-solid regions and the motion of the solidification front can be much faster than differentiation. This prevents differentiation in deep regions. Only a surface region limited by a pressure about 5-10 GPa undergoes a strong differentiation via percolation of the residual melt.

3. A kind of compositional convection occurs due to a layered differentiation. It remixes the differentiating layers but it is weaker than the thermal convection.

4. Remelting due to differentiation is important for deep layers (possibly deeper than $1000 \mathrm{~km}$ ). For the remaining shallow layer of the magma ocean the melt fraction increases to $40 \%$ (at the surface). Further progress in understanding the differentiation and final stratification of the magma ocean requires knowledge of the rheology of partial melts and the kinetics of the crystal growth.

5. The most important chemical implication of the nonfractional crystallization is that a magma ocean could leave almost no traces of its existence in deep layers. The estimates of the composition of the melt and solids in the beginning of differentiation (near the maximum packing crystal fraction) show that at this point, in the entire pressure range, not only the first liquidus solid phase but also subsequent phases have been partially crystallized. Thus any differentiation involves mixtures of minerals. Qualitatively, the degree of differentiation of minor elements could decrease with increase in the degree of crystallization due to equilibration between several coexisting solid phases. It depends, of course, on the partition coefficients. Thus the differentiation could avoid strong differentiation of minor elements resulting from the models of fractional differentiation. Future mass balance calculations can find a solution (a differentiation boundary) which could satisfy both the major and minor element constraints.

Acknowledgments. The authors thank W. Brain Tonks for constructive comments of the first version of the paper and an anonymous reviewer for a discussion of the principal problem. This work was supported by the National Science Foundation grant EAR-89-16611.

\section{REFERENCES}

Abe, Y., Thermal evolution and chemical differentiation of the terrestrial magma ocean, paper presented at the IUGG Symposium on Chemical Evolution of the Earth and Planets, Int. Union Geod. and Geophys., Vienna, 1991.

Abe, Y., Thermal evolution and chemical differentiation of the terrestrial magma ocean, Workshop on the Physics and Chemistry of Magma Oceans from 1 bar to 4 Mbar, LPI Tech. Rep. 92-03, edited by C. B. Agee and J. Longhi, pp. 9-10, Lunar and Planetary Inst., Houston, Texas, 1992.

Agee, C. B., and D. Walker, Mass balance and phase density constraints on early differentiation of chondritic mantle, Earth Planet. Sci. Lett., 90, 144-156, 1988.

Anderson, D. L., Theory of the Earth, Blackwell Scientific, Boston, Mass., 1989.

Anderson, $O$. L., The determination of the volume dependence of the Grüneisen parameter $\gamma, J$. Geophys. Res., 79, 1153-1155, 1974.

Anderson, O. L., R. C. Schreiber, R. C. Liebermann, and N. Soga, Some elastic constant data on minerals relevant to geophysics, Rev. Geophys., 6, 491-524, 1968.

Bell, P. M., T. Yagi, and H. K. Mao, Iron-magnesium distribution coefficients between spinel $(\mathrm{Mg}, \mathrm{Fe})_{2} \mathrm{SiO}_{4}$, magnesiowüstite $(\mathrm{Mg}, \mathrm{Fe}) \mathrm{O}$, and perovskite $(\mathrm{Mg}, \mathrm{Fe}) \mathrm{SiO}_{3}$, Year Book Carnegie Inst. Wash., 78, 618-621, 1979.

Belonoshko, A. B., Equation of state for solid and molten $\mathrm{MgO}$ in high pressure-high temperature range: Molecular dynamics study, Eos Trans. AGU, 73, suppl., 595, 1992.

Boehler, R., Melting of the $\mathrm{Fe}-\mathrm{FeO}$ and the $\mathrm{Fe}-\mathrm{FeS}$ systems at high pressure: Constraints on core temperatures, Earth Planet. Sci. Lett., 111, 217-227, 1992.

Boehler, R., and J. Ramakrishnan, Experimental results on the pressure dependence of the Grüneisen parameter: A review, $J$. Geophys. Res., 85, 6996-7002, 1980.

Bottinga, Y., and D. F. Weill, The viscosity of magmatic silicate 
liquid: A model for calculation, $A m$. J. Sci., 272, 438-475, 1972.

Brinkman, H. C., The viscosity of concentrated suspensions and solutions, J. Chem. Phys., 20, 571, 1952.

Cambell, G. A., and G. Forgacs, Viscosity of concentrated suspensions: An approach based on percolation theory, Phys. Rev., $41 A, 4570-4573,1990$.

Chong, J. S., E. B. Christiansten, and A. D. Baer, Rheology of concentrated suspensions, J. Appl. Polymer Sci., 15, 2007$2021,1971$.

Chopelas, A., and R. Boehler, Thermal expansion measurements at very high pressure: Systematics, and a case for a chemically homogeneous mantle, Geophys. Res. Lett., 16, 1347-1350, 1989.

Cumberland, D. J., and R. J. Crawford, The packing of particles, in Handbook of Pouder Technology, v. 6, edited by J. C. Williams and T. Allen, Elsevier, New York, 1987.

Drake M. J., D. C. Rubie, and E. A. McFarlane, Mgperovskite/silicate melt and magnesiowüstite/silicate melt partition coefficients for KLB-1 at 250 kbars, Workshop on the Physics and Chemistry of Magma Oceans from 1 bar to 4 Mbar, LPI Tech. Rep. 92-09, edited by C. B. Agee and J. Longhi, pp. 17-18, Lunar and Planetary Inst., Houston, Texas, 1992.

Dullien, F. A. L., Porous Media: Fluid Transport and Pore Structure, Academic, San Diego, Calif., 1979.

Fei, Y., S. K. Saxena, and A. Navrotsky, Internally consistent thermodynamic data and equilibrium phase relations for compounds in the system $\mathrm{MgO}-\mathrm{SiO}_{2}$ at high pressure and high temperature, J. Geophys. Res., 95, 6915-6928, 1990.

Frankel, N. A., and A. Acrivos, On the viscosity of a concentrated suspension of solid spheres, Chem. Eng. Sci., 22, 847$853,1967$.

Golitsyn, G. S., Simple theoretical and experimental study of convection with some geophysical applications and analogies, J. Fluid Mech., 95, 567-608, 1978.

Gong, Z. X., and R. E. Cohen, Molecular dynamics simulations of melting of $\mathrm{MgO}$ at high pressures up to $150 \mathrm{GPa}$ Eos Trans. AGU, 72, suppl., 436, 1991.

Heinz, D. L., and R. Jeanloz, Measurement of the melting curve of $\mathrm{Mg}_{0.9} \mathrm{Fe}_{0.1} \mathrm{SiO}_{3}$ at lower mantle conditions and its geophysical implications, J. Geophys. Res., 92, 11,437-11,444, 1987.

Herzberg, C., Phase equilibria and trace element partitioning in a magma ocean to 260 kilobars, Workshop on the Physics and Chemistry of Magma Oceans from 1 bar to 4 Mbar, LPI Tech. Rep. 92-03, edited by C. B. Agee and J. Longhi, pp. 22-23, Lunar and Planetary Inst., Houston, Texas, 1992.

Herzberg, C., and T. Gasparik, Garnet and pyroxenes in the mantle: A test of the majorite hypothesis, J. Geophys. Res., 96, 16,263-16,274, 1991.

Huppert, H. E., R. C. Kerr, J. R. Lister, and J. S. Turner, Convection and particle entrainment driven by differential sedimentation, J. Fluid Mech., 226, 349-369, 1991.

Ito, E., and E. Takahashi, Melting of peridotite at uppermost lower-mantle conditions, Nature, 928, 514-517, 1987.

Ito, E., and H. Yamada, Stability relations of spinels, ilmenites and perovskites, in High Pressure Research in Geophysics: Advances in Earth Planetary Science, edited by S. Akimoto and M. H. Manghnani, pp. 405-419, Cent. Acad. Publ. Jpn., Tokyo, 1982.

Jeanloz, R., and T. J. Ahrens, Equations of state of $\mathrm{FeO}$ and $\mathrm{CaO}$, Geophys. J. R. Astron. Soc., 62, 505-528, 1980.

Jeanloz, R., and Y. Sato-Sorensen, Hydrostatic compression of $\mathrm{Fe}_{1-\mathrm{x}} \mathrm{O}$ wüstite, J. Geophys. Res., 91, 4665-4672, 1986.

Jones, J. H., C. J. Capobianko, and M. J. Drake, Estimation of high temperature metal-silicate partition coefficients, Workshop on the Physics and Chemistry of Magma Oceans from 1 bar to 4 Mbar, LPI Tech. Rep. 92-03, edited by C. B. Agee and J. Longhi, pp. 20-22, Lunar and Planetary Inst., Houston, Texas, 1992.

Kato, T., and M. Kumazawa, High pressure effect on the melting relation in the system $\mathrm{Mg}_{2} \mathrm{SiO}_{4}-\mathrm{MgSiO}_{3}$ : Phase transitions in the constituent phases and differentiation by melting in the Earth's mantle, in Dynamic Processes of Material Transport and Transformation in the Earth's Interior, edited by F. Marumo, pp. 277-308, Terrapub, Tokyo, 1990.

Kato, T., A. E. Ringwood, and T. Irifune., Experimental determination of element partioning between silicate perovskites, garnets and liquids: Constraints on early differentiation of the mantle, Earth Planet. Sci. Lett., 89, 123-145, 1988 a.

Kato, T., Ringwood, A. E., and T. Irifune, Constraints on element partition coefficients between $\mathrm{MgSiO}_{3}$ perovskite and liquid determined dy direct measurements, Earth Planet. Sci. Lett., $90,65-68,19886$.

Knittle, E., and R. Jeanloz, Synthesis and equation of stste of $(\mathrm{Mg}, \mathrm{Fe}) \mathrm{SiO}_{3}$ perovskite to over 100 gigapascals, Science, 235, 668-670, 1987.

Knittle, E., and $\mathrm{R}$. Jeanloz, Melting curve of $(\mathrm{Mg}, \mathrm{Fe}) \mathrm{SiO}_{3}$ perovskite to $96 \mathrm{GPa}$ : Evidence for a structural transition in lower mantle melts, Geophys. Res. Lett., 16, 421-424, 1989.

Knittle, E., and R. Jeanloz, The high-pressure phase diagram of $\mathrm{Fe}_{0.94} \mathrm{O}$ : A possible constituent of the Earth's core, J. Geophys. Res., 96, 16,169-16,180, 1991.

Knittle, E., R. Jeanloz, and G. L. Smith, Thermal expansion of silicate perovskite and stratification of the Earth's mantle, Nature, 319, 214-216, 1986.

Krieger, I. M., and T. J. Dougherty, A mechanism for nonNewtonian flow in suspensions of rigid spheres, Trans. Soc. Rheol., 3, 137-152, 1959.

Landau, L. D., and E. M. Lifshitz, Statistical Physics, Pergamon, New York, 1980.

Lee, D. J., Packing of spheres and its effect on the viscosity of suspensions, J. Paint Technol, 42, 579-587, 1970.

McBirney, A. R., and T. Murase, Rheological properties of magmas, Annu. Rev. Earth Planet. Sci., 12, 337-357, 1984.

McFarlane, E. A., and M. J. Drake, Element partitioning and the early thermal history of the Earth, in Origin of the Earth, edited by N. E. Newsom and J. H. Jones, pp. 135-150, Oxford University Press, New York, 1990.

McFarlane, E. A., M. J. Drake, and T. Gasparik, Mgperovskite/silicate melt partition coefficients in the CMS system at $2430^{\circ} \mathrm{C}$ and 226 kbars, Workshop on the Physics and Chemistry of Magma Oceans from 1 bar to 4 Mbar, LPI Tech. Rep. 92-03, edited by C. B. Agee and J. Longhi, pp. 34-35, Lunar and Planetary Inst., Houston, Texas, 1992.

McGeary, R. K., Mechanical packing of spherical particles, J. Am. Ceram. Soc., 44, 513-522, 1961.

McKenzie, D., The generation and compaction of partially molten rocks, J. Petrol., 25, 713-765, 1984.

McKenzie, D., and M. J. Bickle, The volume and composition of melt generated by extension of the lithosphere, J. Petrol., 29, 625-679, 1988.

Miller, G. H., E. M. Stolper, and T. J. Ahrens, The equation of state of a molten komatiite, 1 , Shock wave compression to 36 GPa, J. Geophys. Res., 96, 11,831-11,848, 1991 a.

Miller, G. H., E. M. Stolper, and T. J. Ahrens, The equation of state of a molten komatiite, 2, Application to komatiite petrogenesis and the hadean mantle, J. Geophys. Res., 96, $11,849-11,864,1991 b$.

Mooney, M., The viscosity of a concentrated suspension of spherical particles, J. Colloid. Sci., 6, 162-170, 1951.

Murray, J. D., On the mathematics of fluidization, Part I, Fundamental equations and wave propagation, J. Fluid Mech., 21, 465-493, 1965.

Murthy, V. R., Early differentiation of the Earth and the problem of mantle siderophile elements: A new approach, Science, 253 , 303-306, 1991.

Murthy, V. R., Evidence for a high temperature differentiation in a molten Earth: A preliminary appraisal, Workshop on the Physics and Chemistry of Magma Oceans from 1 bar to $4 \mathrm{Mbar}$, LPI Tech. Rep. 92-03, edited by C. B. Agee and J. Longhi, pp. 28-29, Lunar and Planetary Inst., Houston, Texas, 1992.

Ohtani, E., Melting temperature distribution and fractionation in the lower mantle, Phys. Earth Planet. Inter., 33, 12-25, 1983.

Ohtani, E., The primordial terrestrial magma ocean and its implication for stratification of the mantle, Phys. Earth Planet. Inter., $38,70-80,1985$.

Ohtani, E., Ultrahigh pressure melting and chemical heterogeneity in the deep mantle, in Dynamic Processes of Material Transport and Transformation in the Earth's Interior, edited by F. Marumo, pp. 253-275, Terrapub, Tokyo, 1990.

Ohtani, E., and H. Sawamoto, Melting experiment on a model chondritic mantle composition at $25 \mathrm{GPa}$, Geophys. Res, Lett., 14, 733-736, 1987.

Ohtani, E., T. Kato, and H. Sawamoto, Melting of a model chondritic mantle to $20 \mathrm{GPa}$, Nature, 322, 352-353, 1986.

Poirier, J. P., Lindemann law and the melting temperature of 
perovskites, Phys. Earth Planet. Inter., 54, 364-369, 1989.

Ringwood, A. E., Phase transformations and the constitution of the mantle, Phys. Earth Planet. Inter., s, 109-135, 1970.

Ringwood, A. E., Earliest history of the Earth-Moon system, in Origin of the Earth, edited by N. E. Newsom and J. H. Jones, pp. 101-134, Oxford University Press, New York, 1990.

Roscoe, R., The viscosity of suspensions of rigid spheres, Br. J. Appl. Phys., 3, 267-269, 1952.

Scarfe, C. M., and E. Takahashi, Melting of garnet peridotite to $13 \mathrm{GPa}$ and the early history of the upper mantle, Nature, 922, 354-356, 1986.

Shaw, H. R., Rheology of basalt in the melting range, J. Petrol., 10, 510-535, 1969.

Solomatov, V. S., Parameterization of temperature- and stressdependent viscosity convection and the thermal evolution of Venus, in Flow and Dynamic Modeling of the Earth and Planets, University of Alaska, Fairbank, in press, 1993.

Solomatov, V. S., and D. J. Stevenson, Suspension in convective layers and style of differentiation of a terrestrial magma ocean, J. Geophys. Res., this issue (a).

Solomatov, V. S., and D. J. Stevenson, Kinetics of crystal growth in a terrestrial magma ocean, J. Geophys. Res., this issue (a).

Soo, S. H., Fluid Dynamics of Multiphase Systems, Blaisdell, Waltham, Mass., 1967.

Stishov, S. M., Entropy, disorder, melting, Sov. Phys. Uspekhi, 31, 52-67, 1988.

Stixrude, L., and M. S. Bukowinski, Fundamental thermodynamical relations and silicate melting with implications for the constitution of $D^{\prime \prime}, J$. Geophys. Res., 95, 19,311-19,325, 1990.

Takahashi, E., and E. Ito, Melting phase relations of mantle peridotite up to $25 \mathrm{GPa}$ : Speculations on the origin and evolu- tion of the Earth's mantle, in Dynamic Processes of Material Transport and Transformation in the Earth's Interior, edited by F. Marumo, pp. 253-275, Terrapub, Tokyo, 1990.

Takahashi, E., and C. M. Scarfe, Melting of peridotite to $14 \mathrm{GPa}$ and the genesis of komatiite, Neture, 315, 566-568, 1985.

Taylor, G. J., and M. D. Norman, Evidence for magma oceans on asteroids, the Moon, and Earth, Workshop on the Physics and Chemistry of Magma Oceans from 1 bar to 4 Mbar, LPI Tech. Rep. 92-03, edited by C. B. Agee and J. Longhi, pp. 58-65, Lunar and Planetary Inst., Houston, Texas, 1992.

Tonks, W. B., and H. J. Melosh, The physics of crystal settling and suspension in a turbulent magma ocean, in Origin of the Earth, edited by N. E. Newsom and J. H. Jones, pp. 151-174, Oxford University Press, New York, 1990.

Turcotte, D. L., and G. Schubert, 1982, Geodynamics: Applications of Continum Physics to Geological Problems, John Wiley, New York, 1982.

Walker, D., and C. Agee, Partitioning "equilibrium", temperature gradients, and constraints on Earth differentiation, Earth Planet. Sci. Lett., 96, 49-60, 1989.

Zindler, A., and S. R. Hart, Chemical geodynamics, Annu. Rev. Earth Planet. Sci., 14, 493-571, 1986.

V. S. Solomatov and D. J. Stevenson, Division of Geological and Planetary Sciences, California Institute of Technology, Pasadena, CA 91125.

(Received May 5, 1992; revised September 18, 1992; accepted October 28, 1992.) 